\title{
L'epistolario Morgagni-Réaumur alla Biblioteca Civica di Forlì
}

\author{
A cura di Luigi Belloni
}

Il carteggio fra Giovanni Battista Morgagni (1682-1771) e René-Antoine Ferchault de Réaumur (1683-1757) offre alla storiografia della scienza un documento perspicuo del processo di divergente specializzazione che accompagnò il rigoglioso sviluppo della biologia promosso dal metodo sperimentale.

Attraverso una indagine globale del mondo organico (animale e vegetale) Marcello Malpighi (1628-94 $)^{1}$, il biologo per eccellenza della Scuola Galileiana ${ }^{2}$, aveva riconosciuto nell'organismo vivente una minuta strutturazione meccanica. Nel mondo animale - che qui particolarmente ci interessa - egli aveva seguito il progressivo complicarsi delle strutture, sia nel corso della embriogenesi, sia nel corso di quella che sarà detta evoluzione filogenetica. Secondo la sua dottrina, continuamente suffragata da reperti di laboratorio, le strutture, più semplici, dell'embrione illuminano quelle, più complesse, dell'animale a termine di sviluppo; e analogamente, le strutture, più semplici, degli insetti illuminano quelle, più complesse, degli animali superiori e dell'uomo.

La comprensione delle strutture normali è inoltre illuminata dallo studio delle strutture abnormi, o comunque alterate, secondo un indirizzo che impartì sostanziali impulsi alla indagine anatomica delle sedi e delle cause delle malattie, per parafrasare il titolo dell'opera in cui esso trovò il suo coronamento: il De sedibus et causis morborum per anatomen indagatis, pubblicato dal Morgagni nel 1761, dopo un sessantennio di indagini anatomiche specificamente dedicate all'uomo e ai vertebrati.

Se in questo campo primeggiò il Morgagni, il suo contemporaneo Réaumur primeggiò, invece, nella indagine entomologica, e non soltanto grazie ai Mémoires pour servir à l'histoire des insectes (1734/42), ma anche agli

${ }^{1}$ È da vedere, in proposito, l'introduzione al volume M. MALPIGHI, Opere scelte a cura di L. Belloni, Torino 1967; H.B. Adelmann, Marcello Malpighi and the evolution of embryo$\log y$, Ithaca (N.Y.) 1966.

${ }^{2}$ Sui primi, e primari, interessi biologici della Scuola Galileiana è da vedere L. BELLonI, La dottrina della circolazione del sangue e la Scuola Galileiana 1636-61, Gesnerus 28 (1971) 7-34. 
stimoli impartiti ad altrui ricerche e scoperte, a quelle, in particolare, sulla partenogenesi degli Afidi (Bonnet) e sulla rigenerazione del polipo d'acqua dolce (Trembley).

La divergenza delle strade percorse dai due scienziati - entrambi continuatori dell'opera del Malpighi - risalta in tutta la sua evidenza nelle parti del carteggio in cui l'anatomo-patologo Morgagni e l'entomologo Réaumur si palleggiano l'un l'altro la competenza nello studio degli insetti e vermi anatomicamente rinvenuti nell'organismo dell'uomo e di animali superiori.

La relazione fra i due studiosi era stata mediata da Sauveur-François Morand (1697-1773) ${ }^{3}$. Entrato nel 1708 quale allievo geometra all'Académie Royale des Sciences, il Réaumur ${ }^{4}$ legò alla medesima la sua feconda e proteiforme attività scientifica e diresse l'illustre sodalizio nello stesso anno $1731^{5}$ in cui il Morgagni ${ }^{6}$ venne chiamato a ricoprire uno degli 8 posti di membro associato straniero.

3 Appartenne alla Académie Royale des Sciences quale «associé ordinaire», per l'anatomia, e fu chirurgo di larga fama. Autore di più lavori sulla litotomia (quello, ad es., citato alla nota 39), egli si rivolse al Morgagni, tramite Pietro Antonio Michelotti (1673-1740), per avere notizie circa l'attività litotomica svolta a Padova nel 1708 da Frère Jacques de Beaulieu (Baulot, Baulieu, 1651-1719).

Il Morgagni rispose in data 2 Gennaio 1732 con lo scritto «De vesicae calculis a Fr. Jacobo Beaulieu Patavii exsectis, et de casu Corneliae Bandiae epistola», Opuscula Miscellanea 1 (Venezia 1763) 31-32.

4 Sul Réaumur, oltre ai numerosi articoli raccolti nella Revue d'Histoire des Sciences et de leurs applications 11 (1958), è da vedere: J.ToRLAIs, Réaumur d'après des documents inédits, Paris 1961.

5 J.Torlais, Chronologie de la vie et des œuvres de René-Antoine Ferchault de Réaumur, Revue d'Histoire des Sciences et de leurs applications 11 (1958) 1-12.

${ }^{6}$ Il quale dedicherà alla Académie Royale des Sciences il $3^{\circ}$ dei 5 libri De sedibus (1761).

7 In proposito, è da vedere: M. Chiadini, Terza serie di carteggi inediti del Morgagni, Società Italiana di Storia delle Scienze mediche e naturali. Atti del III Congresso Nazionale, Venezia 1925, Siena 1926, 146-153.

Brani relativi alle «lunettes en loupe» furono editi da L. MessedagLia, L' «Iter Italicum Patavinum» di D. Cotugno. G.B. Morgagni e l'Università di Padova nel 1765, Atti d. R. Istituto Veneto di Scienze, lettere ed arti 73/2 (1913/4) 1691-1803 (cfr.1761-1764). Il Messedaglia auspicò l'edizione del «prezioso carteggio » fra il Morgagni e il Réaumur, come, d'altronde, la «completa, stampa di tutto il carteggio, edito ed inedito, del Morgagni». 
Le 21 lettere $^{7}$ (11 del Morgagni al Réaumur ${ }^{8}$ e 10 del Réaumur al Morgagni $^{9}$ ) che vengono qui pubblicate ${ }^{10}$ dal Fondo Morgagni della Biblioteca Civica di Forlì ${ }^{11}$, si possono suddividere in 2 gruppi:

$1^{\circ}$ gruppo (Lettere 1-13: dal 1735 al 1743), caratterizzato da periodiche riaccensioni coincidenti con l'uscita di ciascuno dei 6 tomi (1734-6-7-8; 1740-2) dei Mémoires del Réaumur, che l'autore inviava di volta in volta in omaggio al Morgagni, il quale ricambiò con la sua opera Valsalviana (1740). L'epistolario è aperto in latino dal Morgagni; ma viene poi continuato nella lingua-madre di ciascuno dei due corrispondenti. Emergono fra i temi trattati: le «lunettes en loupe $»,{ }^{12}$ l'annuncio delle scoperte del Bonnet e del Trembley, il dibattito fra anatomo-patologo ed entomologo circa le rispettive competenze in campo parassitologico.

$2^{\circ}$ gruppo (Lettere 14-21: dal 1749 al 1752), caratterizzato dai nuovi interessi ornitologici del Réaumur: incubazione artificiale (1749 e 1751), digestione gastrica degli uccelli (1752), museo ornitologico. Da parte del Morgagni va, invece, segnalata l'edizione completa delle lettere su Celso e Samonico (1750).

${ }^{8}$ Vol.15, cc.6-10. Si tratta di minute, interamente autografe del Morgagni. Nell'intento di facilitare il Lettore, l'editore si è arbitrato di apportare alcuni «a capo».

9 Vol.25, cc.4-27. Tutte le lettere sono interamente autografe del Réaumur. L'edizione è stata quindi condotta col maggior rispetto possibile, risparmiando anche le iniziali minuscule dopo i punti. Per l'uso degli «a capo» valga quanto si è detto nella nota 8 .

10 In vista di una edizione sistematica dell'epistolario Morgagnano, alla quale ho già contribuito coi seguenti saggi: Aus dem Briefwechsel von G.B. Morgagni mit L. Schröck und J.F.Baier, Nova Acta Leopoldina 36 (1970) Nummer 198, 107-139; Lettere del 1761 fra D. Cotugno e G.B.Morgagni, Physis 12 (1970) 415-423; Contributi all'epistolario Boerhaave-Morgagni. L'edizione delle «Epistolae anatomicae duae», Leida 1728, Physis 13 (1971) 81-109.

Ricordo inoltre l'opuscolo: G.B.MorgagNI, Gli inventori anatomici italiani del XVI secolo nel carteggio col medico milanese Bartolomeo Corti, a cura di L.Belloni, Milano 1953.

11 Alla quale porgo i più vivi ringraziamenti, e in particolare al Dr. Walter Vichi, Direttore, al Dr. Vincenzo Mezzomonaco, Vice-Direttore, nonchè ai Signori Claudio Albonetti e Luciano Vasumini, che hanno allestito un prezioso, e ancora inedito, inventario dell'epistolario Morgagnano.

Lettere del Morgagni al Réaumur, o viceversa, non risultano conservate negli archivi della Académie des Sciences (per cortese comunicazione dei Segretari perpetui Louis de Broglie e Robert Courrier), nè alla Biblioteca Civica della Rochelle (per cortese comunicazione di M.lle O.de Saint-Affrique, Conservateur).

12 Si veda l'Appendice. 


\section{1.-Morgagni a Réaumur, 7 Settembre 1735}

A Monsieur/Monsieur de Reaumur/de l'Académie Royale des Sciences de/Paris Experientissimo ac Celeberrimo Viro/D. ${ }^{\text {no }}$ D. De Reaumur/Ioannes Baptista Morgagnus S.P.D.

Iam diu est, Vir Celeberrime, ex quo in Commentariis Regiae istius Scientiarum Academiae quidquid potissimum a Te est ${ }^{13}$, id omne incredibili cum voluptate nec minori cum utilitate perlegere solitus sum, propterea quia nec maiorem in excogitando solertiam, neque in experiendo diligentiam, neque in narrando perspicuitatem optari posse crediderim quam qua a Te video, quamcumque rem tractandam suscipias, hane quoad eius natura patitur, in aperto poni, et supra quam sperari posse videbatur, in publicum commodum promoveri. Non ut Modestiae Tuae gravis sim haec commemoro, sed ut facilius intelligas quam mihi gratum fuerit eximium Munus quo me pro Tua singulari Humanitate honestare voluisti, Voluminis Tui Primi ad Insectorum Historiam attinentis ${ }^{14}$. In hoc enim ea omnia suspicio quae supra laudabam, eaque cum tanto numero accuratissimarum tum observationum tum delineationum coniuncta, ut sine ulla dubitatione sperem, hanc difficillimam latissimeque patentem Naturalis Historiae partem a Te uno eò adductam iri quo vel multorum consociata opera facile adduci non posset. Itaque simul gratias ago, et habeo maximas, simul oro, ut strenue, quod facis, pergas, et quoniam me Tibi non communibus tantum, sed et peculiaribus beneficiis obstringere voluisti, hoc quoque addas, ut si qua unquam in re me Tibi inservire posse credas, utaris ut Tuo; nam ut caetera mihi desint, obsequium certe et gratus erga Te animus deesse nunquam poterunt. Vale Vir Doctissime, et mihi, quod fecisti, et facis, favere perge.

D.Patavii VII.Idus Septembr. M DCG XXXV.

\section{2.- Réaumur a Morgagni,13 Giugno 1736}

a paris ce $13^{\mathrm{e}}$ juin 1736

vous avez si bien recu, Monsieur, le premier volume des Memoires sur les insectes que j'en dois etre enhardi a vous envoier le second volume de ces memoires ${ }^{15}$; ce sera assurement sa faute s'il ne vous paroist pas digne des eloges que vous avez donnés au premier, car la disposition favorable ou vous estes pour moy peut vous en donner beaucoup a l'indulgence. vous avez pourtant acquis une si grande habitude de bien juger, qu'il est difficile que les defauts, qui se trouvent sans doute dans cet ouvrage, echapent a des yeux aussi eclairés que les votres. je vous ai dejà prié de m'apprendre ceux que vous aurez trouves dans le premier volume et c'est pour les deux volumes que je vous fais la meme priere. je souhaiterois fort que les volumes qui paroitront fussent de meilleurs en meilleurs. il ne tiendra

13 Utile è, in proposito, la cronologia del Torlais qui citata alla nota 5.

14 Mémoires pour servir à l'histoire des insectes. Par M. de Réaumur, de l'Académie Royale des Sciences. Tome premier: Sur les Chenilles et sur les Papillons, à Paris, de l'Imprimerie Royale, 1734.

15 Tome Second: Suite de l'Histoire des Chenilles et des Papillons; Et l'Histoire des Insectes ennemis des Chenilles, Paris 1736. 
qu'a vous que cela soit: personne n'est plus capable que vous de remetre dans le bon chemin ceux qui s'en ecartent. c'est aussi avec la plus parfaite estime et un tres respectueux attachement que j'ai l'honneur d'etre

Monsieur

Votre tres humble et

tres obeisant serviteur

de Reaumur

\section{3.- Morgagni a Réaumur, 8 Novembre 1736}

a Monsieur/Monsieur de Reaumur/Membre de l'Academie Royale des Sciences/a Paris

Monsieur

Vi scrivo nella mia lingua non tanto perchè sempre più comprendo dalle vostre Opere che ben la intendete, quanto perchè vorrei pure esprimervi meglio che mi sia possibile sì le obbligazioni mie nel riceverle, come il piacere e l'ammirazione nel leggerle. Ma sono astretto a confessarvi ingenuamente che nè meno in italiano sò trovare espressioni, che in questo caso mi bastino. Pur vi dirò, che vi sono tanto obbligato de' vostri Doni, quanto gli stimo: e gli stimo tanto, che non conosco alcuno che nella Naturale Istoria me ne possa far de' maggiori. In fatti quanto gran parte di essa faccian gl'Insetti, quanto utile, quanto maravigliosa, non l'hò sinora meglio compreso, che in leggendo i vostri Libri.

$\mathrm{Ne}$ ' quali mentre così spesso sostituite alle false ed antiche, vere e nuove maraviglie, oh quante altre io ne trovo di queste nella vostra Perspicacità, ò in dedurre giustissimamente l'ignoto dal noto, ò in rinvenire la chiara, e spesso unica maniera di porlo sotto gli occhi di tutti, e questa tanto più facile, quanto alla prima più sembrava difficile, anzi pure impossibile. Allora è, credetemi, che io provo insieme il maggior piacere, e la maggior maraviglia. È tuttavia grande ancor queilla ch'è dovuta alla vostra insuperabile diligenza nell'osservare, singular cautela nel sospendere talvolta il giudizio, ed amabilissima ingenuità nel confessare tal'altra volta, che quella cosa l'avete osservata e che quest'uso non lo sapete. E voi poi trasportato dalla vostra Modestia, e Benignità a me ricorrete quasi dubitaste di non essere sulla buona strada, ò io la potessi conoscere meglio di voi!

Certamente ò io non la conosco punto, ò cotesta che voi tenete è la migliore di tutte. I vostri Tomi susseguenti per esser'ottimi basta che sieno somiglianti ai due primi: cosa assai difficile ad ogni altro che a voi. Così pure io fossi qual la mercè vostra vi piace di credermi perchè potessi vicendevolmente corrispondere a questi due vostri Stimatissimi Doni. Ma se in ciò mi mancheranno le forze; non mi mancheranno giamai, come da prima vi scrissi, ed ora vi confermo, nè un vivo sentimento di gratitudine, nè quel pieno ossequio che si deve ad un Uomo del Grado, e Merito Vostro, e con cui mi dò l'Onore di rassegnarmi

Monsieur

Padova, 8. Novembre 1736.

Vostro umilissimo, ed obbligatissimo Servidore

Giambattista Morgagni. 


\section{4.- Réaumur a Morgagni, 1 Agosto 1737}

a paris ce $1^{\mathrm{er}}$ aoust 1737 .

\section{Monsieur}

connoissant, comme je fais, tout le prix de vos eloges je ne scaurois rien souhaiter de plus au troisieme volume des Memoires sur les insectes ${ }^{16}$, que d'être digne de ceux que vous avez bien voulu donner aux deux premiers. mais je scay qu'il n'est que trop ordinaire aux autheurs des ouvrages de longue haleine de faire paroitre des suites qui repondent mal a ce que les commencements promettoient. le genie se refroidit pour un objet qu'on a depuis trop longtemps sous les yeux; il arrive a l'amour qu'on avoit pour un ouvrage, ce qui arrive a un amour d'une toute autre nature, il s'afoiblit sans qu'on s'en appercoive; et c'est un cas dans lequel j'aurois bien pu etre, sans le scavoir, par rapport a ce troisieme volume; en cas que je vous paroisse etre tombé dans l'assoupissement je vous supplie donc de vouloir bien m'en retirer.

nos memoires de l'Academie, Monsieur, vous auroient ete envoies regulierement a mesure qu'ils paroissent, j'aurois pris avec plaisir ce soin, si je n'avois pas cru que M.Morand en etoit chargé ; mais s'il lui arrivoit d'oublier de receuillir pour vous ce qui paroitra de nouveau dans l'Academie, je ne manquerai pas de l'en faire souvenir. permettez moy, Monsieur, de me servir de cet occasion pour vous faire remarquer qu'il ne tiendroit qu'a vous d'enrichir les productions d'une compagnie qui a pour vous une si grande estime, ce seroit de lui envoier quelques unes de vos dissertations pour etre inserées parmi celles qu'elle publie chaque année. il vous seroit apparemment plus commode de les envoier en italien; ou en latin qu'en francois, mais assez de gens parmi nous s'offriroient a les traduire, et a revoir les traductions. en mon particulier je saisirai toujours avec empressement les occasions propres a vous donner quelqu'idée du devouement parfait, et respectueux avec lequel j'ai l'honneur d'etre

Monsieur

Votre tres humble et

tres obeisant serviteur

de Reaumur.

\section{5.-Morgagni a Réaumur, 2 Giugno 1738}

a Monsieur/Monsieur de Reaumur/de l'Académie Royale de Sciences et Commandeur/et Intendant de l'Ordre Royal et Militaire de S.Louis./a Paris

\section{Monsieur}

Quanto è più ordinario difetto dell'Opere voluminose, che ai bei principj non corrisponda il progresso, tanto è maggiore il pregio di cotesta vostra, il di cui terzo Tomo non cede punto a' due così belli, e così utili che l'hanno preceduto. Io l'hò letto con lo stesso, anzi

16 Tome Troisième: Histoire des Vers mineurs des feuilles, des Teignes, des fausses Teignes, des Pucerons, des ennemis des Pucerons, des faux Pucerons, et l'histoire des Galles des plantes, et de leurs Insectes, Paris 1737. 
con maggior piacere che gli altri due, sì perchè la lettura di quelli mi hà reso più abile a gustare le bellezze di questo, sì perchè m'hanno interessato le lodi da voi date a' miei Italiani, anzi pure a' miei Amici, che tali furono sinchè vissero il Vallisnieri ${ }^{17}$, e il Patarol ${ }^{18}$, per nulla dire de' due, che tanto stimo, Malpighi ${ }^{19}$, e Redi ${ }^{20}$, il primo de' quali sempre più conosco essere parimente in sommo pregio appresso di voi, e l'altro forse da nissuno fù mai lodato più che da voi in quel luogo medesimo in cui giustamente un gravissimo error di lui condannate ${ }^{21}$.

Che dirò poi d'altre cose di mio particolar genio, e di mia propria utilità ?

per cagion d'esempio dove agli altri usi, da me notati, di quella materia oliosa, che il

17 Antonio Vallisnieri (1661-1730), allievo del Malpighi, professore di medicina all'Università di Padova, autore di importanti scritti entomologici, che sono facilmente reperibili nei 3 volumi di Opere fisico-mediche stampate e manoscritte del Kavalier Antonio Vallisneri raccolte da Antonio suo figliuolo, Venezia 1733.

Il Morgagni intende qui riferirsi alla pag. 438 del Tomo $3^{\circ}$ dei Mémoires del Réaumur, come egli stesso indica sul margine esterno della carta $7 \mathrm{r}$ col numero 438 , sotto il quale sono incolonnati i numeri di alcune pagine $(225,475,73,487)$ che verranno richiamate nelle note successive.

18 Lorenzo Patarol (1674-1727). Un suo «Elogio» è da vedere nel Giornale de' Letterati d'Italia 38/2 (1733) 44-63.

Le sue Osservazioni intorno alla Nascita, vitto, costumi, mutazioni, o sviluppi della Cantaride de' Giglj (Dalla Villa di Sandono, li 29. Giugno 1712.) furono inserite dal Vallisnieri nelle Esperienze ed osservazioni intorno all'Origine, Sviluppi, e costumi di varj Insetti, con altre spettanti alla Naturale, e Medica Storia, Padova 1713, 195-222 e 2 Tavv. (cfr. anche A. VAllisneri, Opere fisico-mediche 1, 255-266 e Tavv. XXXII-XXXIII). Il Réaumur cita le Osservazioni del Patarol nei Mémoires 3, 225.

Il Morgagni aveva contratto amicizia col Patarol durante il periodo di permanenza a Venezia (1707/9) e aveva con lui partecipato a osservazioni botaniche: «quo cum et in eius horto, et in veneto littore plantas interdum, dum Venetijs fuit, osbservavit». Così leggiamo nell'autobiografia del Morgagni a c.6v del ms. Ashb. 227/6 della Biblioteca Mediceo-Laurenziana in Firenze, cfr. anche G.B.MoRgagnI, Opera postuma 1, Roma 1964, 27.

19 Nel De bombyce (Londra 1669) il Malpighi aveva riconosciuto l'intima struttura del corpo dell'insetto durante le diverse fasi della sua metamorfosi; e nella Anatomes plantarum pars altera (Londra 1679) aveva negato la generazione spontanea degli insetti delle galle. Queste furono pertanto concepite come il prodotto di una deviazione di sviluppo indotta nel vegetale dalla femmina ovopositrice. Al capitolo «De gallis» di questa seconda opera si riferisce, per l'appunto, il Réaumur nei Mémoires 3, 418.

${ }^{20}$ Francesco Redi (1626-97), autore delle Esperienze intorno alla generazione degli insetti, Firenze 1667. Cfr. L.Bellon,, Francesco Redi, biologo, Celebrazione dell' Accademia del Cimento nel tricentenario della Fondazione (Domus Galilaeana, 1957), Pisa 1958, 54-70; Francesco Redi als Vertreter der italienischen Biologie des XVII. Jahrhunderts, Münchener medizinische Wochenschrift 101 (1659) 1617-1624.

21 Mémoires 3, 4.75. 
Valsalva, ed io chiamammo sebacea ${ }^{22}$, aggiugnete con tanta evidenza quello di conservare e ne' vivi animali, e dopo morte ancora, le lane ${ }^{23}$,

ò dove insegnate pelle più minute notomie a valersi d'occhiali non men forti de' micro$\operatorname{scopj}^{24}$, cosa tanto necessaria, che mille volte hò desiderato d'aver tre mani, per tenere con l'una il microscopio, con l'altra l'oggetto, e per incidere con la terza; onde mi perdonerete se vi pregherò a volermi dire (con vostro comodo però, e in occasione di farmi l'Onore di scrivermi per comandarmi) come precisamente sien fatti, e quanto ingrandiscano cotesti tali occhiali de' quali voi vi servite, e come ve ne serviate, e se senza che la vista a lungo andare ne provi danno.

Per questi adunque, e per altri somiglianti motivi; se vi hò molto, come ben dovea, ringraziato del primo, e del secondo Tomo della vostra eccellente Opera; assai più vi ringrazio del terzo.

Nè minori grazie vi rendo delle profferte obbligantissime di cui vi degnate favorirmi intorno ò al farmi ottenere que' Tomi delle Memorie di cotesta Dottissima Reale Accademia delle Scienze che di mano in mano andranno uscendo, ò all'invigilare perche fosse ben tradotta in francese qualche Dissertazione ch'io mandassi alla medesima. Verrà forse il tempo che io vi supplichi di questa Grazia con quella fiducia che mi porge la Somma Cortesìa vostra quando mi sentirò avere non già cose degne di una così Grande Accademia ${ }^{25}$; che non le spero, ma un po' di respiro da un lungo, e difficil lavoro in cui ancora per qualche anno mi trovo così impegnato ${ }^{26}$ che ad altra applicazione non posso per alcun modo dividermi. Voi intanto Signore conservatemi, vi prego, la vostra Stimatissima Grazia, onoratemi de' vostri Comandi, e credete che alle molte obbligazioni che vi professo, corrispondono perfettamente l'alta stima ed il sommo ossequio con cui mi fò gloria di essere

Monsieur

Padova 2. Giugno 1738.

22 La denominazione risale ad Antonio Maria Valsalva (1666-1723) nel De aure humana, Bologna 1704, p.3: «Tales glandulas», ossia quelle da lui per la prima volta ritrovate nella cute del padiglione auricolare, "Glandulas Sebaceas dicam».

Delle ghiandole sebacee il Morgagni si era già occupato negli Adversaria anatomica prima (Bologna 1706) e alle medesime dedicherà la Epistola anatomica III (Venezia 174.0,T.I, pp.1-60, cfr.qui Nota 50) nel quadro di un ampio dibattito sulla dottrina della ghiandola, che ricorre, sia pure di sfuggita, anche nell'epistolario Boerhaave-Morgagni qui citato alla nota 10 (cfr.la Fig. 3).

23 Mémoires 3,73.

${ }^{24}$ Cfr. l'Appendice.

${ }_{25}$ Tramite il Morand, il Morgagni comunicherà all'Académie R. des Sciences alcuni reperti anatomici di Conformations singulières dei quali è fatto un breve cenno nella Histoire de l'Académie Royale des Sciences. Année MDCCXLI (Paris 1744) 76-77.

Dedicando nel 1761 il $3^{\circ}$ libro De Sedibus all'Accademia medesima, il Morgagni afferma esplicitamente di voler riparare alla mancata collaborazione: «... neque ego nisi obsequiosi animi officiis unquam potuerim eximiae illi Vestrae erga me Liberalitati respondere, quippe hic assiduis occupationibus districtus meis ...».

${ }^{26}$ L'opera Valsalviana, che apparirà a Venezia nel 1740 (cfr. la nota 50 ). 


\section{6.- Réaumur a Morgagni, 21 Dicembre 1738}

a paris ce $2^{\mathrm{i}}$ decembre 1738.

Monsieur

faisant autant de cas que j'en fais de votre estime, je devrois avoir moins d'empressement que j'en ai de vous faire parvenir le $4^{\mathrm{e}}{ }^{\mathrm{v}}$ volume des Memoires sur les insectes ${ }^{27}$, car je ne scaurois esperer qu'il vous paroisse digne de touts les eloges que vous avez donnés au volume qui l'a precedé. si cependant je suis encore a repondre a la letre que vous m'avez fait l'honneur de m'ecrire le 2 juin, a cette letre remplie de tant d'oblijeants temoignages, c'est que j'ai cru etre en etat de vous envoyer beaucoup plutot, l'exemplaire de ce $4^{\mathrm{e}}$ volume que divers contretemps ne m'ont permis de vous adresser qu'aujourdui.

l'amour de la verité m'a forcé d'y dire en quelques endroits ${ }^{28}$ que M. Vallisnieri n'a pas ete toujours aussi exact qu'il a coutume de l'etre, et que j'eusse souhaité qu'il l'eut été.

je ne scay, Monsieur, si vous vous accommoderez aussi bien que je le fais de lunettes qui grossoient beaucoup les objets, qui peuvent tenir lieu de loupe ${ }^{29}$. il faut quelque habitude a s'en servir, et si vous n'estes pas dans la nescesité d'avoir recours pour lire aux lunettes ordinaires, l'habitude de vous servir des autres vous sera plus difficile a acquerir. mais je ne crois pouvoir mieux faire que de vous envoyer une paire de ces lunettes pour que vous en fassiez l'essay. elles vous serviront de modelle pour celles que vous voudrez vous faire faire avec des verres d'un plus court, ou d'un plus long foyer; car il faut en avoir de rechange. je ne puis decider si votre vue se trouvera ou ne se trouvera pas fatiguee par l'usage de pareilles lunettes. pour moy je suis fort content de la mienne, quoique toute ma vie je n'aye cessé de regarder de petits objets autravers des fortes loupes, ou des microscopes. a la verite je me sers de lunettes ordinaires pour lire depuis quelques annees, mais j'en rejette la cause sur la flamme des fourneaux que j'ai regardee sans assez de precaution pendant beaucoup d'autres années ${ }^{30}$.

vous ne m'avez point marqué, Monsieur, si vous recevez regulierement le volume de nos memoires de l'Academie qui paroist chaque année, il vous appartient de droit. je souhaite voir arriver le temps que vous me lassiez esperer, ou libre d'un travail qui vous occupe actuellement, vous nous envoyerez des dissertations qui surement enrichiront nos memoires; et c'est ce que le vif interest que je prens a tout ce qui peut contribuer a la gloire de l'Academie ne me permet pas de souhaiter foiblement. j'ai l'honneur d'etre avec la plus grande estime, et un devouement tres respectueux

Monsieur

Votre tres humble et

tres obeisant serviteur

de Reaumur

27 Tome quatrième: Histoire des Gallinsectes, des Progallinsectes, et des Mouches à deux aîles. Afferma il Réaumur a p.iij «...nous commençons ce volume par l'histoire des insectes, qui ont été pris eux-mêmes par d'habiles Naturalistes, pour des galles de plantes et d'arbres, pour des portions d'écorce ou de bois. Nous leur avons donné le nom de Gallinsectes, propre à faire entendre que, quoiqu'ils ressemblent à des galles, ils sont réellement des insectes.» 


\section{7.- Morgagni a Réaumur, non datata}

... des Sciences, de celle de Petersbourg etc. et Commandeur...

Benche il quarto Tomo delle vostre essattissime, ed utilissime Memorie sopra gl'Insetti molto sia stato per viaggio; è però qualche mese che avrei potuto, come ben dovea, e come ora fò, rendervene infinite grazie, se prima non avessi voluto anche in mezzo alle mie in parte già scrittevi occupazioni, leggerlo tutto attentamente. Vi assicuro con la mia solita naturale ingenuità, che il piacere, l'ammirazione, ed il numero delle cose che hò imparate in leggendolo, non è stato punto minore che ne' Tomi precedenti: nè dubito, che se il Sig. General Marsigli ${ }^{31}$, ò il Sig. Vallisnieri vivessero, non fossero pronti a riconoscere, che voi avete invincibil ragione, ed a ringraziarvi insieme della maniera obbligantissima con la quale dissentite da loro; avvegnachè mi ricordi che il primo, che pur'era mio Compare, ed Amico, non era troppo contento di quella ritenutezza dell'altro che voi avete bene osservata alla pag. $47 .^{32} \mathrm{ma}$ in fine poi al vero, messo come da voi, in tutto il suo lume, sò che l'animo filosofico del Sig. ${ }^{\mathbf{r}}$ Generale non avrebbe fatta la minima resistenza.

${ }^{28}$ Quelli relativi ai Gallinsetti del Kermes saranno messi particolarmente in rilievo dal Morgagni nella Lettera 7 (cfr. la nota 32).

${ }^{29}$ Cfr. l'Appendice.

${ }^{30} \mathrm{Nel}$ corso delle sue ricerche tecnologiche. Si veda, ad es., A. Birembaut, Réaumur et l'élaboration des produits ferreux, Revue d'Histoire des Sciences et de leurs applications 11 (1958) 138-166.

${ }^{31}$ Luigi Ferdinando Marsili (1658--1730), che fornì raccolte e mezzi per la fondazione dell'Istituto delle Scienze di Bologna. La lettera del Marsili al Vallisnieri intitolata Annotazioni intorno alla Grana de' Tintori detta Kermes e datata Bologna 7 Marzo 1711, apparve in appendice al Brieve ristretto del saggio fisico intorno alla Storia del Mare (Venezia 1711, pp.53-72 e 2 Tavole) dello stesso Marsili.

${ }^{32}$ Per comprendere il brano del Réaumur cui il Morgagni si riferisce, va tenuta presente la Istoria della grana del Kermes, e di un'altra nera Grana, che si trova negli Elici delle campagne di Livorno, de' Moscherini spurj della medesima, delle Cimici degli Agrumi, de' Pidocchi de' Fichi, ... di Giacinto Cestoni (1637-1718), datata Livorno 20 Settembre 1714 e indirizzata al Vallisnieri, che la pubblicò, con una sua prefazione, in Opere diverse del Sig. Antonio Vallisnieri, Venezia 1715, pp.161-180 e 8 tavv. (donde fu ristampata in A. VALlisneri, Opere fisico-mediche 1, 457-465 e tavv. XLV-XLVIII; e in G. CestonI, Epistolario ad Antonio Vallisnieri con introduzione ed a cura di Silvestro Baglioni, 2, Roma 1941, 714-730).

Nella suddetta Istoria il Cestoni assimila la grana del kermes ai Gallinsetti degli elici, degli aranci e dei fichi. Dice appunto il Réaumur a p. 47: «M. Vallisnieri a fait imprimer parmi ses œuvres cette Lettre de M. Cestoni, qu'il a fait précéder par une courte Préface, dans laquelle il rappelle ce qu'on avoit écrit jusqu'alors sur le kermès. Quoique personne ne fût un plus grand juge que lui sur de pareilles matiéres, il a semblé n'oser prononcer en faveur du sentiment de M. Cestoni, il a laissé au public la liberté entiére de se déterminer. J'ai pourtant peine à croire que M. Vallisnieri n'ait pas bien vû toute la force des inductions de M. Cestoni, qui approche de la démonstration, mais j'ai bien du penchant à penser que sa politesse pour M. le Comte de Marsilli l'a retenu. Il n'a pas 
Il mio non ne fà già alcuna alle osservazioni ${ }^{33}$ che proponete alla pag.419. e seguenti, perchè son vostre, cioè fatte non solo tante volte, ma con tanta cautela ancora, attenzione, ed avvedutezza, quanta ben vedo in leggendole, tuttavia le cose mi paiono singulari a tal segno che non le crederei facilmente ad altro Osservatore, che a voi, alla cui diligenza, e giudizio tanto deferisco che qualunque dubbio in contrario l'hò per effetto d'una prevenzion mal fondata.

Negl'Insetti, che la mia Professione m'hà più volte messi sotto gli occhj, cioè ne' vermi degl'intestini e d'altre Parti interne dell'uomo, e degli animali v'hà molte cose, sulle quali non resto pago nè secondo l'altrui, nè secondo le mie quali si siano osservazioni. Oh quanta speranza avea posta nelle vostre ricerche, rissoluto di stare a ciò che da voi fosse determinato. Ma se hò bene intesa quest'ultima vostra Prefazione ${ }^{34}$, temo assai, che questa sorte d'Insetti con tutta la sua varietà, e con tutto il vantaggio che dal ben conoscerli potrebbe risultarne alla Medicina, e per conseguenza al genere umano, cosa per altro da voi sommamente ricercata, non resti indietro: il che, s'è possibile, vi prego, e supplico quanto sò, e posso, a far che non segua. Il Genio vostro benefico, e generoso mi dà fiducia.

Io vi pregai d'alcune notizie semplicemente intorno agli occhiali che ingrandiscono gli oggetti $^{35}$ : e la vostra Liberalità me gli hà mandati, belli, e perfettissimi. Ve ne rendo quelle grazie che posso maggiori; ma insieme vi prego d'accennarmi qualche maniera onde tutta la mia gratitudine non resti sempre nell'animo, e nelle parole. Io non hò alcun vizio tanto in abborrimento, quanto l'ingratitudine, e la menzogna. Per altro sino a questa età di cinquantotto anni, io leggo ancora senza occhiali ${ }^{36}$ : tuttavia non diffido d'avezzarmi col tempo a far buon' uso del vostro, a me gratissimo, Dono.

Mons. Morand ${ }^{37}$ che mi hà favorito di farmi avere le vostre Stimatissime Grazie, mi favorisce ancora per quelle di cotesta Regia Accademia, e già n'ebbi l'ultimo Tomo dell' $A$. $1735^{38}$. Io vi prego, che in vedendolo vogliate farmi l'Onore d'accertarlo che già ricevei i

voulu se déclarer ouvertement contre le sentiment que ce dernier avoit cherché à établir dans une Dissertation qu'il lui avoit adressée à lui-même», alludendo con ciò alle Annotazioni da noi citate alla nota 31 .

33 Sull'anatomia dell'addome gravido dei Ditteri.

${ }^{34}$ Con particolare riferimento alle pp. xxvj-xxviij, in cui il Réaumur annuncia il programma dei prossimi volumi.

L'appello del Morgagni è ancora più comprensibile per chi abbia letto la $12^{\mathrm{a}}$ memoria dello stesso tomo $4^{\circ}$ : «Des mouches à deux aisles qui ont l'air de bourdons; et de la mouche du ver du nez des moutons» (pp.497-572 e tavv.34-38).

${ }^{35}$ Si veda l'Appendice.

36 N.Scalinci, Gli occhi, i mali d'occhi e la deficienza visiva degli ultimi anni di Giovan Battista Morgagni, Atti e memorie dell'Accademia di Storia dell'Arte Sanitaria 22 (1956) $58-69$.

37 Cfr.la Nota 3.

${ }^{38}$ Dal frontespizio il volume della Histoire de l'Académie Royale des Sciences... avec les Mémoires... per il 1735 risulta stampato nel 1738. 
vostri Doni insieme con la di lui ben giusta Confutazione di quel luogo di M. Sharp ${ }^{39}$, della quale molto il ringrazio. Nel libro intitolato Connoissance des Temps ${ }^{40}$, ch'egli pure mi manda, hò osservato che alla pag. 206. dove è segnato il mio povero nome ${ }^{41}$, si potrebbe secondo l'uso aggiungervi ${ }^{42}$ ciò che noterò quì sotto, non perchè io sia vago di titoli; che se il fossi, mi basterebbe per tutti quello d'Accademico delle Scienze; ma perchè ad altri non paia forse ch'io faccia poca stima di quelli onde per loro somma Benignità, non per mio merito alcuno, ben vollero onorarmi. Tuttavia questo sia detto confidentemente a voi solo, acciochè, se vi par bene, ne facciate uso, come di vostro proprio moto: se nò ; il poniate per non detto. Sono con la più grande stima, obbligazione, ed ossequio

Docteur en Medecine, et Premier Professeur d'Anatomie dans l'Université de Padouë, de la Societé Royale de Londres, et de celle de Petersbourg, etc. à Padouë.

\section{8.- Réaumur a Morgagni,13 Agosto 1740}

a paris ce $13^{\mathrm{e}}$ aoust 1740 .

je n'avois pas cru, Monsieur, que je serois plus d'un an sans vous remercier de votre derniere et tres obligeante letre; j'avois pourtant compté differer ma reponse jusques a ce que je pusse l'accompagner d'un exemplaire du $5^{\mathrm{e}}$ volume ${ }^{43}$ des memoires sur les insectes. mais j'avois lieu de croire que l'impression de ce volume seroit finie cinq' a six mois plutot qu'elle ne l'a eté. l'imprimerie Royale dont l'allure est souvent fort lente, a redoublé de lenteur. je me consolerai du retardement si vous le lisez avec quelque plaisir.

dans ce volume comme dans les autres il n'y a que les faits que j'ai vus, et de la maniere dont je dis les avoir vus. j'aurois ete charmé que vous eussiez eu occasion d'observer vous meme ceux que j'ai rapportés ${ }^{44}$ dans le $4^{\mathrm{e}}$ tom.pag. 419 en vous assurant de leur certitude

${ }^{39}$ Il Morgagni allude all'opera del Morand (sul cui interesse per la litotomia è da vedere la nota 3) intitolata: Réfutation d'un passage du Traité en Anglois, publié par M. Sharp, chirurgien de Londres, sur la taille latérale, Paris 1739. Il chirurgo londinese Samuel Sharp (1700-78) è autore di un celebre trattato di operazioni chirurgiche (A treatise on the Operations of Surgery), apparso a Londra nel 1739, che ebbe una decima edizione nel $1782 \mathrm{e}$ fu anche tradotto in francese (Parigi 1741).

40 Connoissance des temps pour l'année 1739. au meridien de Paris, publiée par l'ordre de l'Academie Royale des Sciences, et calculée par M.Maraldi, de la même Academie, Paris $1739,206$.

${ }^{41}$ Fra gli otto «Associez étrangers» è indicato al $7^{\circ}$ posto «M. Morgagni, Docteur en Medecine, et Professeur d'Anatomie dans l'Université de Padouë, à Padouë».

Identica qualifica del Morgagni ricorre anche nell'annata 1740, sempre a p.206, della Connoissance des temps.

42 Come, infatti, avvenne a partire dall'annata 1741 della Connoissance des temps, p.210.

43 Tome cinquième: Suite de l'Histoire des Mouches à deux aîles, et l'Histoire de plusieurs Mouches à quatre aîles, sçavoir, des Mouches à scies, des Cigales, et des Abeilles, Paris 1740.

${ }^{44}$ Cfr. la Nota 33. 
vous eussiez probablement saisi quelques circonstances, qui m'ont echapé et qui eussent aidé a lever les difficultes qui vous font peine. mais il faut avouer que les machines animales des insectes ont eté faites sur des principes differents de celles des autres animaux.

j'avois soupconne par exemple dans l'histoire des pucerons, qui se trouve dans le $3^{\mathbf{e}}$ volume ${ }^{45}$, qu'ils se multiplioient sans accouplement. j'ai invite a faire les observations propres a apprendre ce qu'on en doit penser; un jeune homme de geneve ${ }^{46}$, qui a toute la patience, et les autres qualites nescesaires a un bon observateur, a pris un puceron dans le moment ou il venoit de naitre, il l'a fait croitre sous ses yeux dans la plus parfaite solitude, toujours couvert d'un vase de verre dans les moments ou il ne le veilloit pas. et au bout de dix a douze jours ce puceron, qui n'avoit eu commerce avec aucun de ceux de son espece a mis au jour des petits vivants.

je ne suis pas plus content que vous, Monsieur, de tout ce qui a ete publié jusques ici sur les differentes especes de vers du corps humain. de les bien connoitre ne seroit pas un objet d'une simple curiosite; mais malgre tout le desir que j'aurois de m'instruire de leur histoire, et quoique le desir que j'en avois naturellement ait ete rendu plus vif, par vos pressantes invitations, je dois desesperer d'y pouvoir travailler. les occasions de les etudier me manquent ; ce n'est qu'un medecin qui peut les avoir; et surtout un medecin qui diseque beaucoup de cadavres. mais qui peut mieux decouvrir ce que l'histoire de ces vers a de plus important, que vous Monsieur, qui examinez continuellement l'interieur des corps humains avec des yeux si eclairés, et si attentifs. personne ne peut mieux remplir cette tâche, et elle digne de vous.

vous trouverez dans la connoissance des temps de 1740 ; a votre article, les additions qui y devoient etre ${ }^{47}$. les memoires de $1737^{48}$ paroissent depuis plusieurs mois, et l'impression des memoires de $1738^{49}$ est avancee.

j'ai l'honneur avec la plus grande estime, et un parfait et respectueux attachement

Monsieur

Votre tres humble et

tres obeisant serviteur

de Reaumur

\section{9.- Morgagni a Réaumur, 20 Dicembre 1741}

Sicome non dubito che voi, mio Signore, avrete saputo da Mons. Morand, che non prima di questi ultimi mesi m'è pervenuto il Tomo quinto delle vostre incomparabili Memorie sopra gl'Insetti, il quale sin dall'Agosto del 1740. vi compiaceste di consegnarli per me,

${ }^{45}$ Cfr.pag. 329.

${ }^{46}$ Le scoperte di Charles Bonnet (1720-93) sulla partenogenesi degli Afidi forniranno materia al Réaumur per inserire nel $6^{\circ}$ tomo (1742, pp. 523-68 e tav.47) una $13^{\text {a }}$ memoria intitolata: "Addition à l'histoire des pucerons, donnée dans le troisième volume ( $3^{\mathrm{e}}$ Mém.), Sur la manière dont ils se multiplient».

47 Cfr. le Note 40 e 41.

${ }^{48}$ Dal frontespizio il volume della Histoire accademica per il 1737 risulta stampato nel 1740.

${ }^{49} \mathrm{Il}$ cui volume risulta esso pure stampato nel 1740. 
unito ad una vostra obbligantissima lettera; così spero che non vi sarete maravigliato del non aver' io sino ad ora risposto a questa, e rese a voi quelle grazie che posso maggiori sì dell'uno, come dell'altra. Il fò ora con tutto l'ossequio, e con tanto maggior debito, quanto più dalla lettura d'amendue s'è accresciuta in me la stima, che avea per altro infinita, sì del Merito, e Valore, sì della Benignità, e Cortesìa vostra. Quando voi non aveste scritto intorno agl'Insetti, se non quanto in questo ultimo Tomo ci avete dato dell'Api; tanto bastava per far comprendere quanta sia la vostra essattezza nell'osservare, quanto l'ingegno nel trovar modi di vedere, e certificarsi di cose le più riposte, e difficili, quanta la saviezza nell'inferire dalle vostre osservazioni, e quanta l'erudizione e l'equità nel giudicar delle altrui, quanta la disposizione, e la chiarezza nello scrivere, e rappresentare, e quanta finalmente la premura del pubblico bene, che non lasciate mai veruna occasion di promuovere non meno con tante nuove osservazioni, che con tante utili riflessioni.

E voi poi nella vostra Gentilissima lettera non solamente vi prendete la pena d'assicurarmi benignamente della certezza de' fatti da voi riferiti, e delle maravigliose differenze che tra gl'insetti, e gli altri animali s'incontrano; ma in oltre con la più obbligante maniera m'invitate ad intraprender l'istoria de' vermi del corpo umano! Le vostre Memorie per se stesse quanto m'assicurano della verità, che manifestamente spicca e risplende in ogni parte anche la più sorprendente delle medesime, altre tanto mi spaventano dall'impresa, facendomi conoscere tutto quel che mi manca per ben riuscirvi. Di pazienza, e d'amore sì del vero, sì del pubblico vantaggio credo d'essere assai ben fornito. Ma di tanti altri requisiti non meno necessarj, quali sono quelli appunto che di sopra hò lodati, mi conosco assai men proveduto di quel che si ricercherebbe, e che io bramerei. A questo s'aggiunga una cosa ugualmente vera ed importante, ma forse più manifesta, cioè l'inesperienza mia nello studio difficilissimo degl' insetti, per cui non m' hànno lasciato mai tempo le continue mie occupazioni in quello del corpo umano, e degli altri animali, che dal volgo perfetti si chiamano. Volendo, potrete facilmente certificarvene dalle poche, e tanto imperfette mie osservazioni intorno a'vermi dell'uno, e degli altri, inserite in quelle mie Epistole sopra il Valsalva ${ }^{50}$ che mi diedi l'onore di farvi presentare umilmente, non perchè le credessi degne di voi, ma solamente per darvi segno, che se avessi cose migliori, tanto più volontieri ve le offrirei in testimonio dell'ossequio, della stima, e della gratitudine che in sommo grado vi professo. Scorrendole in qualche momento de' meno occupati con la facilità che l'Indice somministra, vedrete s'io dico il vero: e beato me, se da quelle prendeste motivo d'illuminarmi dove mi troverete dubitare, ed errare, e più beato, se prendeste quello di riosservare co' que' gran lumi che avete, alcuni di que' vermi almeno, che ne' cani, ò negli uccelli, ò ne' pesci ho veduti. Sò di certo che tali cose e in essi, e nella maniera d'essaminarli, c'insegnereste, che assai men difficile poi sarebbe a noi altri Medici l'avanzare in questa parte d'Istoria, che per nostra disgrazia voi ci lasciate. Ma questo sia detto per confermarvi la necessità che avremmo, che voi almeno la cominciaste; non per distogliervi dal primo proponimento vostro, nè dal compire l'edizione di quelle Memorie, che non solo i Medici, ma tutti gli amatori della naturale Istoria che tanto e con tanta giustizia, applaudiscono alle già pubblicate, aspettano, anzi impazientemente desiderano.

${ }^{50}$ Le Epistolae anatomicae duodeviginti ad Scripta pertinentes Celeberrimi Viri Antonii Mariae Valsalvae integrano, con l'edizione Morgagnana delle opere del Valsalva, i due tomi Viri celeberrimi Antonii Mariae Valsalvae Opera ..., Venezia 1740. 
Resta che vi renda, come fò con tutto lo spirito, grazie ancòra dell'aggiunta fatta fare al mio articolo dans la Connoissance des Temps ${ }^{51}$ : e vi preghi che vi piaccia prima di perdonarmi della ristretta carta sù cui involontariamente vi scrivo, poscia di far' avere l'inclusa a Mons. Morand; ma sopra tutto di credermi quale mi glorio di essere con la maggiore stima, e col più sincero perfettissimo ossequio

Monsieur

Padova 20. Dec. ${ }^{\mathrm{e}}$ 1741.

Vostro umilissimo ed obbligatissimo servitore

10.- Réaumur a Morgagni,1 Aprile 1742

a paris le $1^{\text {er }}$ avril 1742 .

Monsieur

M. Morand qui scavoit que j'avois souhaité vous faire parvenir promptement le $5^{\mathrm{e}}$ volume des memoires sur les insectes, a pensé qu'il valoit mieux me laisser ignorer combien de temps il etoit resté en route, que de m'apprendre un retardement qui ne pouvoit que m'etre desagreable; je n'en ai donc ete informé que par votre tres obligeante letre du 20 decembre de l'année derniere. elle m'a confirmé de nouveau dans l'idée que j'avois des dispositions favorables ou vous estes pour moy. je reconnois que je leur dois en grande partie les eloges flatteurs que vous donnez aux memoires de ce dernier volume. si ceux de ces memoires qui traitent des abeilles ont eu l'avantage de vous plaire le fond de la matiere interessante par elle meme, en a ete la principale cause; vous m'aurez d'ailleurs scu gré de m'etre exposé a beaucoup de piquures redoutables. depuis plusieurs mois je me suis promis de vous envoier bientot le sixieme volume de ces memes memoires, mais l'impression a ete plus lente que je ne l'avois prevu, et n'est pas meme encore finie ; et c'est ce qui m'a fait differer a vous ecrire, plus longtemps que je ne l'avois compte;

j'en suis extremement honteux, et je le dois etre surtout de ne vous avoir pas encore fait mes remerciments des deux volumes ${ }^{52}$ que M. Morand me remit de votre part avant les vaccances de l'année derniere; ils ont ete pour moy un present tres precieux. je suis bien eloigné d'etre aussi grand anatomiste qu'il le faudroit etre pour en connoitre toute la valeur, mais il n'est pas hors de ma portee de voir avec quelle sagesse et quelle justesse de raisonnement les differentes matieres qui sont l'objet de vos scavantes letres, y sont discutees; d'y admirer que non seulement vous aiez lu tout ce qui a ete ecrit en anatomie par les anciens et par les modernes, mais que tout ce que les uns et les autres ont publié vous soit aussi present que si vous veniez de lire dans l'instant, et que ce n'est que la nescesité, et le desir de rendre justice a chaque autheur qui vous force a faire usage d'une erudition si immense; partout on y trouve des observations qui n'ont pu etre que le fruit de longues et penibles recherches faites par des yeux trés clairvoyants.

je n'avois pas besoin d'etre invité a lire celles qui sont, sur tout dans votre $9^{\mathrm{e}}$ et dans la $14^{\mathrm{e}}$ letre, sur les vers qui se tiennent dans l'interieur des animaux; je les ai lues, et relues

51 Cfr. la Nota 42.

52 I due tomi citati alla Nota 50. 
et elles m'ont confirmé dans l'idee que j'avois eue que si quelqu'un peut nous donner des lumieres sur l'histoire de ces insectes, c'est assurement vous, Monsieur; ce n'est qu'a des dissections sans nombre et de sujets de differentes especes qu'on peut devoir des observations sur des animaux toujours caches dans l'interieur des autres animaux. les occasions de les voir sont si rares, et on ne les voit en vie que pendant un temps si court, et dans des circonstances si peu favorables, que je crois pourtant qu'il faudra nous resoudre a ignorer pendant longtemps, si ce n'est pas pour toujours, une grande partie des faits les plus essentiels a leur histoire.

nous serons apparemment obliges a en rester pour toujours a des conjectures sur la maniere dont les premiers ont ete deposés dans les corps des animaux plus grands, qui en sont peuplés; il ne me semble pas, qu'ils y aient pu etre conduits par d'autre voye que celle de la circulation. les vers des corps des hommes leurs ont apparemment ete apportes soit par le sang, soit par le lait qu'ils ont tire soit de leurs meres, soit de leurs nourices. les œufs de ces insectes, ou ces insectes naissants peuvent etre d'une petitesse qui leur permet de circuler avec les liqueurs dans nos vaisseaux. il seroit a souhaiter que le microscope put nous faire decouvrir soit dans le sang, soit dans d'autres liqueurs de ces petits œufs, ou de ces petits animaux. des observateurs auxquels je ne me fie pas trop ${ }^{53}$, ont dejà pretendu avoir vu dans le sang des petits animaux de differentes especes. mais nous scavons certainement qu'on en trouve dans la semence, s'ils ne sont pas de petits hommes, ils pourroient bien n'etre que des vers qui apres avoir pris un certain degre d'accroissement, sont portes et deposes dans des parties ou ils achevent de croitre. quoiqu'il en soit de cette derniere conjecture, il n'y a aucune impossibilite a ce que la mere transmette des œufs de vers, ou des vers naissants, a ses enfants; et on ne peut ce me semble imaginer aucune autre voye raisonnable de les transmetre.

Mr. Vallisneri ${ }^{54}$ a très bien fait voir l'absurdite du sentiment de M.Andri ${ }^{55}$, et de ceux

53 Cfr. L. Belloni, Micrografia illusoria e «animalcula», Physis 4. (1962) 65-73; Antonio Vallisneri e il contagio vivo, Il metodo sperimentale in biologia da Vallisneri ad oggi (Simposio nel III ${ }^{\circ}$ Centenario della nascita di Antonio Vallisneri, Padova 1961), Padova 1962, 185-200.

Accanto alle osservazioni illusorie ingenuamente compiute da uomini di scienza, vanno ricordate le mendaci dimostrazioni di Infusori nel sangue e altri liquidi organici da parte di ciarlatani. Cfr. L.Belloni, Charlatans et contagium vivum au déclin de la première période de splendeur de la microscopie, Colloque sur la microscopie et la micrographie aux $X V I I I^{e}$ et $X I X^{e}$ siècles. $85^{e}$ Congrès des Sociétés savantes (ChambéryAnnecy 8-12 avril 1960), Paris 1961, 579-587.

54 Nelle Considerazioni, ed esperienze intorno alla generazione de' vermi ordinarj del corpo umano, Padova 1710; ristampate nelle sue Opere fisico-mediche 1, Venezia 1733,113-178 e Tavv. XVIII-XXI.

Una lettera elmintologica del Morgagni al Vallisnieri, datata 2 Settembre 1708, trovasi inclusa nelle testè citate Considerazioni (pp.132-136; e Opere fis.-med.,pp.173-175) e fu ristampata dallo stesso Morgagni, col titolo «De lumbricis epistola», in Opuscula miscellanea 1 (Venezia 1763) 25-26.

55 Nicolas Andry de Boisregard (1658-1742): De la génération des vers dans le corps de l'homme. De la nature et des espèces de cette maladie, de ses effets, de ses signes, de ses 
qui veulent avec lui que des vers de fruits, de plantes etc. puissent devenir des vers d'animaux.

vos observations prouvent tres bien que la glande thyroide ${ }^{56}$ ne doit pas etre regardee comm'un ovaire de vers, et elles prouvent aussi que des glandes, et d'autres excroissances ${ }^{57}$ peuvent etre habitees par des vers qui ne les ont pas percées pour s'y loger, et qui ce me semble n'ont pu s'y rendre que par la voye de la circulation.

mais peut on esperer de decouvrir si ces vers sont ovipares, ou s'ils sont vivipares! peut etre y en a-t-il des especes des uns, et des especes des autres.

on peut soupconner par exemple que ces vers ronds qui vous ont paru, et qui etoient reellement singuliers, pour la longueur et la finesse de leur queue ${ }^{58}$, sont vivipares; que cette queue etoit un embrion naissant, quelque Bizarre que semble ce soupcon il est fondé sur la maniere dont se perpetue une tres petite espece de millepieds aquatiques ${ }^{59}$, dont j'aurai occasion de parler dans mon dernier volume ${ }^{60}$; sont bout posterieur pousse une espece de queue qui devient de plus en plus longue. cette queue se detache ensuite et est un millepied, qui ne differe que par son volume, du millepied a qui il doit le jour.

vos observations sur differentes especes de vers taenia ${ }^{61}$ prouvent incontestablement qu'il y a aumoins des especes de vers plats, dont la suite des anneaux appartient a un seul et meme animal. mais il ne me paroist pas encore assez etabli qu'il ait de ces chaines qui soient faites d'insectes acroches bout a bout, ceux que j'ai observés etoient surement des animaux composes d'un tres grand nombre d'anneaux. M. Winslou ${ }^{62} \mathrm{~m}$ 'a assuré, et c'est pour moy comme si je l'avois vu, qu'aiant injecté un ver plat, l'injection avoit passe d'un bout a l'autre. le taenia fut il un vray ver solitaire, se trouva $t$ il seul dans le corps d'un animal, la nature pourroit lui avoir donné le moyen de se multiplier, sans avoir besoin de s'accoupler avec un ver de son espece; elle pourroit avoir fait pour lui ce qu'elle a fait pour les pucerons, qui sembloient en avoir moins besoin car il est a present demontré, et vous en trouverez les preuves les plus certaines dans le $6^{\mathrm{e}}$ volume des memoires sur les insectes $^{63}$, que les pucerons de plusieurs especes sont en etat de metre des petits au jour sans avoir ete fecondés; que tenus dans la plus parfaite solitude depuis le moment de leur naissance jusqu'apres leur derniere transformation; ils accouchent de petits vivants.

prognostics: Des moyens de s'en préserver, et des remèdes pour la guérir etc. Avec trois lettres écrites à l'Auteur, sur le sujet des Vers; les deux premières d'Amsterdam par M. Nicolas Hartsoeker, et l'autre de Rome, par M.Georges Baglivi, Paris $1700 ;$ Eclaircissement sur le livre De la génération des vers dans le corps de l'homme, Paris 1704.

${ }^{56}$ Epistola anatomica IX, § 38 (t.I,pp. 272-273; cfr.la Nota 50).

${ }^{57}$ Epistola anatomica IX, $\S \S 44-46$ (t.I,pp. 279-284).

58 Epistola anatomica XIV, $§ \S 42-43$ e 58 (t.II,pp.45-47 e 60-61).

${ }^{59}$ Allude alla rigenerazione del polipo d'acqua dolce, sulla quale il Réaumur ritornerà più avanti in questa stessa lettera. Cfr. la Nota 64.

${ }^{60}$ Tome sixième,pp.xlix e ss.

${ }^{61}$ Epistola anatomica XIV, $\S ~ 46-57$ (t. II,pp. 46-60).

62 Jacques-Bénigne Winslow (1669-1760), anatomico a Parigi.

${ }^{63}$ Cfr.la Nota 46. 
mais ${ }^{64}$ la nature a eu recours encore a une autre resource pour multiplier et conserver certaines especes d'animaux, dont vous n'avez peutetre pas encore entendu parler, et dont aumoins il ne sera pas inutile que je vous atteste la verite. quand on connoist, Monsieur, aussi bien que vous tout l'appareil prodigieux qui entre dans la composition du plus petit insecte, peut on croire qu'il y en ait qui comme les plantes puissent etre multipliés par boutures! le fait est vray cependant. il y a plusieurs especes d'insectes, et surtout d'insectes aquatiques, des especes de vers qui etant coupés par morceaux se trouvent multipliés. tel ver coupé en huit, dix vingt etc. parties devient huit, dix, vingt etc. vers. un des bouts, l'anterieure repousse une partie posterieure; le bout posterieur repousse une teste et tout cequil faut pour remplacer la partie anterieure. enfin chaque troncon intermediaire pousse par son bout anterieur une tete et par son bout posterieur une queue. vous imaginez bien que le raisonnement n'a pas conduit a faire une pareille decouverte, il ne sembleroit propre qu'a en demontrer l'impossibilité ; elle n'est due qu'au hazard, mais a un de ces hazards qui n'arrivent qu'a ceux qui scavent voir. M. trembley, qui demeure a la haye, en hollande, et qui pretend avoir pris le goust d'observer les insectes dans les memoires que j'ai publiés, me fait le plaisir de me communiquer ce que ces petits animaux lui ont offert de nouveautes remarquables. il y a 15 a 16 mois qu'il m'ecrivit qu'il avoit trouvé dans des eaux un petit corps d'une forme singuliere, qui avoit des mouvements tres lents, et qui le laissoient incertain si ce corps etoit une plante, ou un animal. il n'imagina rien de mieux pour s'assurer de sa nature, que de le couper en deux; comptant que s'il etoit une plante il se multiplieroit dans l'eau, et que s'il etoit un animal il ne tarderoit pas a perir. les deux portions faites par la division devinrent chacune un corps semblable au premier; et on eut des preuves et des moins equivoques que l'un et l'autre des nouveaux petits corps etoient de veritables animaux, d'une espece meme qui est vorace, et qui a des adresses pour prendre des insectes encore plus petits, dont elle se nourrit. j'ai repeté les experiences qu'avoit faites M. trembley sur des insectes de meme genre que le sien et de differentes especes. en fin je les ai tentees sur beaucoup d'especes de differents genres; j'en connois actuellement plus de 20 especes a qui une si etrange proprieté a ete accordee, et je ne suis point revenu encore de la surprise que m'ont causee des reproductions si peu attendues, et si inconcevables.

mais en jugeant par analogie, les especes d'insectes a qui je la connois me disposent a croire que plusieurs especes des vers qui vivent dans l'interieur d'animaux plus grands, ont aussi cette propriete. si elle a ete accordee aux vers plats, tres sujets a se rompre, quand il se fait des reproductions dans deux parties qui ont ete separées, elles peuvent occasionner des changements dans la figure du bout cassé, dont la cause n'eut pas ete facile a deviner.

il seroit a souhaiter qu'on put faire des experiences propres a nous apprendre si les vers des intestins des animaux, sont reellement de ceux qui se reproduisent par boutures. elles demandent beaucoup de circonstances difficiles a trouver; il faudroit tirer des vers bien vivants du corps d'un animal, et apres les avoir coupes par morceaux, les introduire dans

64 A questo punto il Réaumur riprende il discorso (cfr.le note 59 e 60) sulla rigenerazione del polipo d'acqua dolce scoperta da Abraham Trembley (1710-84), che dedicherà alla sua scoperta i Mémoires pour servir à l'histoire d'un genre de polypes d'eau douce, à bras en forme de cornes, Leida 1744. 
les intestins d'un animal de meme espece. ce ne pourroit gueres etre par l'anus, probablement il faudroit ouvrir quelque part les intestins de l'animal, dans les quels on voudroit les loger; et que cet animal restait en vie pendant plusieurs jours malgre l'operation qu'il auroit souferte. peutetre que cette experience reussiroit sur les chiens; mais je ne scay personne qui fut plus propre que vous, Monsieur, a la faire reussir, s'il vous prenoit envie de la tenter. je scay que vous estes capable de surmonter de plus grandes difficultés.

aussi est ce avec la plus grande estime, et le plus parfait devouement que j'ai l'honneur d'etre

Monsieur

\author{
Votre tres humble et \\ tres obeisant serviteur \\ de Reaumur
}

\title{
11.- Réaumur a Morgagni, 30 Dicembre 1742
}

a paris ce 30 decembre 1742 .

les volumes precedents, Monsieur, des memoires sur les insectes ont ete si longtemps, apres etre sortis de chez moy, pour parvenir jusqu'a vous, que j'ai cru devoir tenter une autre voye pour faire tenir celui $\mathrm{ci}^{65} ;$ je scay bien qu'il arrivera a Rome a jour nommé, et je scay encore que l'obligeant pere Mazolleni ${ }^{66}$, a qui je l'adresse, fera tout son possible pour trouver une occasion de le faire partir pour padoue; mais je ne scay pas assez si ces sortes d'occasions sont rares ou frequentes. je voudrois aumoins que ce volume eut le merite de vous etre offert de bonne heure; vous pensez bien que je fais pour lui des souhaits qui vont plus loin, que je desire encore davantage que les observations qu'il contient soient dignes de plaire a un juge aussi eclairé que vous l'estes.

peutetre n'avez vous appris encore que nous avons perdu, il y a 17 a 18 jours, $\mathrm{Mr}$. hunauld ${ }^{67}$, c'etoit un garcon d'esprit, qui aimoit extremement l'anatomie, et capable de travailler utilement pour elle. je le regrette beaucoup.

vous pouvez aussi n'avoir pas scu que les poulmons de Mr. Morand se sont tres mal trouvés de son voiage de flandres; il a ete obligé d'en revenir vers la toutssaint apres avoir craché du pus. je suis assez content de son etat actuel. c'est assurement un grand chirurgien, extremement connu et que la douceur de son caractère fait aimer de touts ceux qui le connoissent. mais pour bien scavoir la structure des poulmons, on ne les en a pas meilleurs. si la connoissance des parties de notre machine mettoit en etat de la faire durer davantage, vous jouiriez d'une vie aussi longue que celle que me font vous souhaiter l'interest que je prens aux progres des sciences, et le parfait et respectueux devouement avec lequel j'ai l'honneur d'etre

Monsieur

\author{
Votre tres humble \\ et tres obeisant serviteur \\ de Reaumur
}

${ }_{65}$ Tome sixième: Suite de l'Histoire des Mouches à quatre aîles, avec un Supplément à celle des Mouches à deux â̂les, Paris 1742 .

${ }_{68}$ Angelo Mazzoleni (1719-68) della Congregazione dell'Oratorio.

67 François-Joseph Hunauld (1701-52), anatomico a Parigi. 


\section{2.- Morgagni a Réaumur, 1 Marzo 1743}

a Monsieur/Monsieur de Reaumur/de l'Académie Royale des/Sciences de Paris, et de celle/de Londres, et de Petersbourg/etc. Commandeur, et Inten/dant de l'Ordre royal et/militaire de S.Louis/a Paris.

Il mio lungo soggiorno lontan da Padova, e il mio tardo ritorno, e le innumerabili distrazioni, che come in sì fatti casi suol'accadere, m'hanno quì sinora occupato, sono state le vere cagioni per le quali prima di ringraziar voi, mio Signore, della savia, e lunga, e perciò tanto a me più utile, e grata vostra lettera del $\mathrm{p}^{0} \mathrm{~d}$ 'Aprile dell'anno scorso, io mi trovi sorpreso da un'altra, non meno obbligante che insieme col dono a me carissimo del Tomo sesto delle vostre eccellenti Memorie sopra gl'Insetti, oggi appunto hò ricevuta per mezzo del Cortesissimo P.Mazzoleni. Per mezzo di lui med. ${ }^{\text {mo }}$ hò voluto subito inviarvi queste poche righe, acciochè sappiate, che da lui sono stato prontamente; e liberalmente favorito; e che sì di questo vostro gentilissimo pensiero sì del libro med. ${ }^{\text {mo }}$ vi sono quanto dir si possa obbligato. Mentre mi riserbo lo scrivervi più a lungo quando avrò letto diligentemente tutto il libro, intanto v'assicuro che fieramente m'è dispiaciuta la perdita importante di M. ${ }^{\mathbf{r}}$ Hunauld ch'io conoscea anche di faccia, e nulla meno ciò che mi accennate del nostro Stimatissimo, ed Amat. ${ }^{\text {mo }}$ M. ${ }^{r}$ Morand quantunque mi ricrei alquanto l'intendere che eravate però assai contento del di lui stato. A Dio piaccia di conservarcelo ben lungamente, ed a voi di fargli sapere, insieme co' miei complimenti, che spero di aver quanto prima quel fagotto da Torino, e che avutolo ; subito gliene scriverò. Sopra tutto, fatemi la giustizia di credermi uno de' più obbligati servidori, e de' più riverenti ammiratori che voi abbiate.

Monsieur

Pad. ${ }^{a}$ 1. Marzo 1743

\section{3.- Morgagni a Réaumur,16 Agosto 1743}

In quella mia breve lettera, che il Gentilissimo P. Mazzoleni mi favorì di mandarvi, mi diedi l'Onore di scrivervi, ch'altra più lunga ve n'avrei inviata quando avessi letto attentamente tutto il sesto Volume delle vostre incomparabili Memorie intorno a gl'Insetti. Ora io non solamente l'hò letto, ma in varie parti l'hò riletto, e più che in ogni altra nella Memoria XIII ${ }^{68}$. e nella Prefazione ${ }^{69}$, la quale, come scrissi ultimamente al nostro amatissimo Mons.Morand, ci hà qui tutti riempiuti d' indicibile maraviglia, e di non minor desiderio di vedere a lungo descritte, ed illustrate con Figure le stupende cose, le quali ivi in breve accennate. Benchè me ne aveste già data cortesemente notizia nella vostra dottissima lettera del primo d'Aprile dell'anno scorso, non lasciarono di sorprendermi in leggendole messe più in vista nella sopradetta Prefazione: or che sarà quando in tutto il loro lume le vedrò esposte dalla vostra eccellente penna, insieme con la maniera più facile di poterne rifar le prove come sarebbe ne' lombrici terrestri. Io voglio allora con le mani, e co gli occhj proprj soddisfarmi a mio potere in questa materia; di cui un'altra più sorpren-

${ }^{68}$ Cfr.la Nota 46.

${ }^{69}$ Cfr.la Nota 60 (e 59). 
dente non mi si è offerta in tutto il tempo della mia vita, nè forse sarà per offrirmisi se non da voi, ò da altri a cui voi abbiate istillato il buon gusto nell'osservare gl'Insetti, fonte inesausto di maraviglie. Di verità di tanto rilievo le quali la sola povera umana ragione non ci avrebbe mai condotti a trovare, anzi ce ne avrebbe come voi stesso confessate facilmente dissuasi, ne abbiamo noi, e ne avranno i posteri tutto l'obbligo ò la maggior parte alle vostre Memorie. Ed io nel mio particolare v'hò quello in oltre d'aver ricevuti molti bei lumi dalla mentovata lettera vostra, mercè de' quali sento destarmisi nuove idee sopra quelle mie poche, e deboli osservazioni, che con tanta Bontà non solo vi prendeste la pena di leggere, ma voleste ancora far degne delle vostre savissime riflessioni.

Veramente altre strade non sò vedere ancor'io che quelle del latte, e del sangue per trasportare da un corpo all'altro, e da una parte all'altra i piccoli vermi, ò le loro uova: e se per l'addietro ci avea alcune difficoltà, non ne fò più caso da che vedo che non ne fate voi che senza dubbio dall'Istoria degl'Insetti avrete forti argomenti per sorpassarle.

Così quel sospetto vostro intorno a quella quasi coda di tanta lunghezza e sottigliezza in que' vermi tondi da me descritta, come fondato sulla nuova elegante osservazione che ci promettete di certi millepiedi acquatici, mi fà ardentemente bramare che a suo luogo intieramente e con le Figure ce la esponiate per farne io allora più accertato confronto con que' miei vermi.

Così intorno alle Tenie, oltre la bella osservazione del Celeb. ${ }^{\text {mo }}$ Mons. Winslow per provare che ve ne siano di quelle che non sono che un verme solo, convincentissimi sono gli esempj d'altri animali per far capire che quando ancora una, e non più, se ne trovasse in un' animale; essa ben potrebbe senza accoppiarsi esser feconda, ò moltiplicarsi e riprodursi a foggia delle piante troncate, come fanno tanti animali di cui nella già detta Prefazione scrivete. E senza dubbio questa seconda maniera quanto conviene alle Tenie esposte in molte occasioni al pericolo del rompersi, tanto è comoda come pur voi saggiamente avvertite per render ragione di certe varietà che nelle medesime spesso incontriamo. E se mi verrà fatto di poter aver qualche cane che col dar fuori alcuna di esse, mi faccia congetturare che forse ne abbia dell'altre io son disposto a tentare l'esperienza per altro assai difficile da riuscire e, senza replicarla in diversi cani e con certi contrasegni, anche ambigua, che da voi cortesemente mi vien suggerita.

Per le quali cose tutte, quanto sò e posso e di quella pregiatissima vostra lettera, e di questo non men bello degli altri Volume sesto, grandemente vi ringrazio. In ciascuna delle Memorie ch'esso contiene ritrovo tutte le qualità che hò ammirate ne' precedenti: e benchè la XIII ${ }^{\mathrm{a}}$. abbia in oltre, come hò di sopra accennato, molto di sorprendente; non ne manca però nell'altre e segnatamente nella XIV. ${ }^{a}$ e nella XII. ${ }^{a}$ la quale offre massimamente a noi Italiani uno spettacolo quanto più insolito, tanto più bello ${ }^{70}$.

E perchè la vostra Modestia non abbia ad attribuire, come già fece nel proposito dell'Api, la mia principale ammirazione al fondo medesimo della materia; io posso con sincerità dichiararvi che cotesto fondo non giungerei di gran lunga a comprenderlo se da voi non fosse gia esaminato, emendato, accresciuto, illustrato, il che voi facendo, mi trovo sorpreso ugualmente, per non dir più, sulle materie note, che sulle ignote, e potrei darvene più d'un'esempio nella Memoria X. ${ }^{a}$

${ }^{70}$ È la «nevicata» di Efemeri descritta a pp.468-469. Si veda anche la vignetta a p.1. 
Ma senza partir dall'Api, occorrendomi ieri l'altro di cercar non sò che nel primo Tomo dell'Apologia che cento e più anni sono, scrivea l'Hofmanno pro Galeno ${ }^{71}$, m'avvenni a caso in queste parole che non posso contenermi dal quì trascrivervi dalla pag. 53. Qui apes vident mellificantes, vel in alvearibus vitreis, vel per foramen ferreis filis clausum, nihil aliud vident, quam apes hinc inde discurrentes stipato agmine: in ipso mellificio admirantur potius Naturae solertiam, quam ut dicere ausint se vidisse, quomodo id factum sit. Che cosa dal principio del mondo è più nota di questa, che l'api facciano il mele ? Sù questo fondo quanti aveano già lavorato per sapere come il facciano anche prima d'osservarle come quegli antichi Romani da voi mentovati ${ }^{72}$, in alvearj trasparenti! Sino al tempo dell'Hofmanno non aveano, anche in alvearj di vetro, veduto nulla. Quel di più che vi s'era veduto innanzi che voi pensaste alle maniere d'obbligar le api a non celarvi alcuno de' loro secreti, ben sapete quanto era poco. Ora se noi sappiamo come facciano il mele; a chi ne abbiamo l'obbligazione? al fondo? ò all'ultimo Coltivatore d'esso fondo?

Dio ci conservi pure questo Coltivatore; che importa troppo la conservazione di questo per conoscere negl'Insetti le più bell'opere della Sapienza, ed Onnipotenza di lui. Quanti Volumi egli dà fuori, tante migliaia di tali cognizioni acquistiamo. Ringrazio Dio, che l'abbia donato al mondo al mio tempo: e ringrazio lui dell'obbligantissima Benignità che si degna d'aver per me, e per le mie deboli produzioni. Riputerò mio grande Onore che la sappiano i posteri, sicome ora è mia gloria che tutti sappiano che sono con non minor obbligazione che ossequio

Padova 16 Agosto 1743.

\title{
14. Réaumur a Morgagni, 6 Settembre 1749
}

a paris ce $6^{\mathrm{e}}$ septembre 1749 .

occupé, Monsieur, comme vous l'estes a tant de recherches difficiles, et qui contribuent tant aux progres de l'anatomie, je ne dois pas imaginer que vous puissiez faire aucun usage des pratiques qui sont enseignees dans l'ouvrage ${ }^{73}$ dont je vous envoye un exemplaire. je me borne a souhaiter que vous le puissiez lire sans ennui. il me fournist une occasion qui m'est precieuse, de vous renouveller les assurances de la tres haute estime, du tres parfait attachement et du respect avec lequel j'ai l'honneur d'etre

Monsieur

\author{
Votre tres humble \\ et tres obeisant serviteur \\ de Reaumur
}

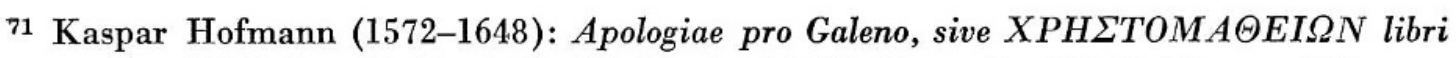
tres... ex Bibliotheca Guidonis Patini, Lugduni 1668, Tomo I, p.53, lib. II., sect. III., cap. X. «De fibris».

72 Mémoires 5, 219.

${ }^{73}$ Art de faire éclorre et d'élever en toute saison des oiseaux domestiques de toutes especes, Soit par le moyen de la chaleur du fumier, soit par le moyen de celle du feu ordinaire, Paris 1749. 


\section{5.- Morgagni a Réaumur, 31 Marzo 1750}

Allo stesso M. ${ }^{\mathrm{x}}$ de Reaumur.

Ciò che mi diedi l'Onore di scrivervi verso il fine d'una mia ben lunga lettera de'16. Agosto 1743. intendo di replicarlo nel rendere che fò ora grazie senza fine alla somma Cortesìa vostra del Dono Pregiatissimo che m'avete fatto dell'Arte di far nascere, e d'allevare in ogni stagione uccelli domestici di tutte le specie. Io quanto più tardi per la mia lunga absenza da Padova l'hò ricevuta insieme con la Gentil. ${ }^{\text {ma }}$ Lettera vostra del 6. Settembre, tanto più avidamente ed attentamente l'hò letta dal principio sino al fine: e posso ingenuamente accertarvi, che dopo avervi imparato un numero grande di cose, che molto mi sarebbe tornato a conto di sapere quando ne' primi anni di questo secolo mi misi a rifare le osservazioni dell'uova covate, altro dispiacere non hò avuto che quello di non aver potuto imparare altre cose importanti da quella Memoria, la quale sul fine dell'Opera ci avete voluto più tosto fare sperare, che darci.

Per altro nel leggere le prime Memorie, meco stesso hò detto più d'una volta, oh quanto godrebbe, se fosse vivo, il nostro Veslingio ${ }^{74}$ in vedere effettuato tanto più ampiamente, $\mathrm{e}$ facilmente di quel che egli sperava, in Europa ciò che egli di vista osservava in Egitto ${ }^{75}$, come abbiamo dalla prima delle Osservazioni di lui pubblicate ${ }^{76}$ dal Bartolini ${ }^{77}$, nella quale ci descrive i forni Egiziani.

Così nel leggere altre Memorie, d'altre cose mi son ricordato, per cagion d'esempio in leggendo la seconda del Tomo II. alla pag. $172 .{ }^{78}$ m'è tornata in mente un'usanza che era già e forse è ancora nella mia Patria, di far portare pollastrini ne' granai quando cominciavano a nascervi farfallette, delle quali e degl'insetti ond'elle provengono, unicamente pascendosi, diventavano essi magri, ma preservavano il rimanente de' grani.

Ma ciò che scrivete nell'ultima Memoria ${ }^{79}$ intorno a quel condotto escretorio ${ }^{80}$ che s'alza dal groppone degli uccelli, m'hà bensì fatto risovvenire di cosa che alcuni quì dicono d'aver provata, cioè che stringendo quel condotto con filo di seta alle piccole pollastre, queste si fanno grassissime; ma se poco io credea prima tal cosa, ora che hò letto quanto dite d'esso condotto, meno ancora la credo.

74 Johann Vesling (1598-1649), professore di anatomia a Padova.

75 Observationes Philosophicae et Medicae de pullorum in Aegypto exclusione mediante calore elementari (datate 6 Maggio 1637).

${ }^{76}$ In Joannis Veslingj Mindani Equitis Observationes anatomicae et epistolae medicae ex schedis posthumis selectae et editae a Th.Bartholino, Copenhagen 1664, pp.1-31.Tali osservazioni del Vesling furono pubblicate dal Bartholin quale appendice alla propria opera De insolitis Partus Humani viis Dissertatio Nova. Accedunt Cl. V. Johannis Veslingi Equitis De Pullitie Aegyptiorum et aliae ejusdem Observationes Anatomicae et Epistolae Medicae posthumae, Copenhagen 1664.

77 Thomas Bartholin (1616-80), anatomico a Copenhagen.

${ }^{78}$ Laddove il Réaumur racconta che una sua amica si era servita dei polli per disinfestare un granaio, dopo aver notato che essi preferivano nutrirsi con gli insetti infestanti, trascurando il grano.

79 «Esquisse des amusemens philosophiques que les oiseaux d'une basse-cour ont à offrir », tomo $2^{\text {a }}$, pp.303-304 (pp. 331-332 della $2^{\text {a }}$ edizione).

${ }^{80}$ Della ghiandola dell'uropigio. 
Tanto basti avervi accennato, perchè sappiate che tutta hò letta l'Opera vostra, tanto bella, e insieme tanto utile al pubblico che nulla più. Ma che è della continuazione dell'altra vostra maggior Opera intorno a gl'Insetti? È gran tempo che eccettuata la sopradetta vostra non hò lettere di Parigi, onde saperne: e quando passò di quì il Sig. Abate Nolet ${ }^{81}$, mi favorì bensì di lasciar quì per me, allora e di poi per molto tempo lontano, l'invoglio che per me gli avea dato Mons. Morand ma nell'invoglio non ci trovai di questo lettera alcuna. Vi prego di fare ad amendue i miei ringraziamenti. Spero di scrivere al secondo in questa prossima estate, se sarà uscita per via delle stampe certa mia piccola $\operatorname{cosa}^{82}$, che qualunque siasi mi darò l'onore di rassegnare a voi, ed a lui. Intanto cogli attestati delle mie sempre maggiori obbligazioni, e dell'altissima stima, e pieno ossequio che vi professo, e professerò sempre, passo a confermarmi

Padova 31. Marzo 1750.

\section{6.- Morgagni a Réaumur,12 Giugno 1750}

Allo stesso M. ${ }^{\mathbf{r}}$ de Reaumur

Facendo io, come devo, mia gloria della Bontà, che un'Uomo di tanto valore, e di tanta stima, come voi siete, si degna d'aver per me, non è da maravigliarsi, che il Sig. ${ }^{\mathbf{r}}$ Dottor Vincenzo Pozzi ${ }^{83}$, da cui presentata vi sarà questa mia riverente lettera, dovendo costà portarsi per continuarvi gli studj suoi, me n'abbia fatta tale istanza che io non hò saputo negargliela. Egli è figlio di Monsignor Pozzi ${ }^{84}$ Camerier Segreto e Medico di Sua Santità, e nipote per Madre del fù Sig. ${ }^{r}$ Pier Jacopo Martelli ${ }^{85}$, amendue Letterati Celebri, e miei sincerissimi Amici: e senza questo pel suo talento, studio, e saviezza che di gran lunga supera l'età, egli è meritevole d'essere da me e da chiunque il conosce, distintamente amato, e raccomandato, come ora lo è da me a voi acciochè volontieri di quel consiglio, e di quell'appoggio gli vogliate far grazia, ch'egli vi chiederà per proseguire sotto ottimi Direttori le sue fisico-mediche applicazioni. Ve ne prego dunque quanto più sò e posso istantemente, accertandovi che con questo nuovo Favore aggiungerete un gran cumulo a tutti gli altri che sinora m'avete benignamente impartiti, e per cui sono non solo con altissima stima, ma con somma obbligazione ancora quale umilmente riverendovi passo a rassegnarmi

Padova 12. Giugno 1750

81 Jean-Antoine Nollet (1700-70).

82 In Aur. Cornelium Celsum et Q. Ser. Samonicum Epistolae decem, quarum sex nunc primum prodeunt, Padova 1750. Queste lettere sono generalmente reperibili nel $2^{\circ}$, e ultimo, tomo dell'opera: A. Cornel. Celsus et Q. Serenus Samonicus, De medicina ... Editio novissima, in qua ad caetera omnia quae in priore nostra, Epistolae sex accedunt Cel.Jo. Bapt. Morgagni nunquam antea vulgatae, Padova 1750.

83 Vincenzo Pozzi (1727-90).

${ }^{84}$ Giuseppe Pozzi (1697-1752). Sulla sua lettera fatta stampare dal Morgagni in appendice alle Epistolae anatomicae duae (Leida 1728), cfr.p.104 dell'epistolario BoerhaaveMorgagni qui citato alla Nota 10.

85 Pietro Jacopo Martelli (1665-1727).

\section{8}




\section{7.- Morgagni a Réaumur,15 Settembre 1750}

Allo stesso M. ${ }^{\mathbf{r}}$ de Reaumur

Eccovi quella piccola, e poco ò nulla pregevol cosa ${ }^{86}$, la quale scrissi ch'era per mandarvi, quando vi ringraziai con una mia de' 31 . Marzo dell'eccellente Opera vostra intorno al far nascere ed allevare uccelli domestici in ogni stagione. Come altro motivo non hò di presentarvi questo mio libricciuolo, se non quello che nulla esca alle stampe del mio, che a voi riverentemente non sia rassegnato; così a me basta che non alla cosa la quale certamente non è degna di venire nelle vostre mani, ma badiate come vi prego unicamente all'animo col qual ve la mando pieno d'altissima stima, di somma gratitudine, e di profondo ossequio, con cui mi glorierò sempre di essere

Monsieur

Padova 15. Sett. 1750.

Vostro umilissimo et obbligatissimo servitore

\section{8.- Réaumur a Morgagni, 3 Aprile 1751}

a paris ce $3^{\mathrm{e}}$ Avril 1751.

\section{Monsieur}

il y a deja pres de deux mois que j'ai recu vos dix letres sur Celse et sur Serenus Samonicus; aussi suis je tres honteux d'etre encore aujourdui a vous en faire mes remerciments. je n'ai pu parvenir a les lire aussitost que je me l'etois promis, et c'est ce qui est cause que j'ai tant tardé a vous en marquer ma reconnoissance. vous donnez le nom de petit livre a celui qu'elles composent, c'en est cependant un qui doit vous avoir coute bien du temps. pour rassembler tout ce qu'elles renferment d'historique, pour parvenir a une critique aussi etendue, et aussi profonde, combien n'a-t-il pas fallu lire d'ouvrages, combien de passages n'a-t-il pas fallu comparer! des remarques solides et judicieuses ont ete le fruit de tant de recherches. j'ai regret de ce que ce fameux et hautain critique Burneman ${ }^{87}$ n'ait pas vecu assez pour voir votre derniere letre, il me semble qu'il en eust du etre bien humilie. elle montre la difference qu'il y a entre un ouvrage de critique fait par un simple grammairien, et celui qui l'est par un philosophe.

le petit mot, Monsieur, que vous m'avez dit en passant dans votre tres obligeante letre du 31 mars 1750 , de vesling ${ }^{88}$, a ete pour moy un avertisement de lire ce qu'il a ecrit sur le fours a poulets d'egypte, dont j'ai profité. on fait actuellement au louvre une seconde edition ${ }^{89}$ de l'art de faire eclorre et d'elever les oiseaux domestiques, dans laquelle vesling dont il n'avoit pas ete fait de mention dans l'autre, sera cité plus d'une fois ${ }^{90}$. je me suis flatte que vous ne desaprouveriez pas que j'y rapportasse ${ }^{91}$ ce que vous m'avez appris de

86 Cfr. la Nota 82.

87 Nella seconda lettera «in Q. Serenum Samonicum», il Morgagni è infatti in polemica con Pieter Burmann (1668-1741), che aveva curato una edizione annotata del De medicina di Q.Serenus Samonicus in Poetae latini minores 2 (Leida 1731) 185-388.

${ }^{88} \mathrm{Si}$ vedano le Note $74-76$.

89 Apparve nel 1751 «à Paris, de l'Imprimerie Royale».

90 Ad es., a pp. 5-6 e 24. del $1^{\circ}$ tomo.

91 A p. 195 del $2^{\circ}$ tomo. 
l'usage ou l'on a ete et ou l'on est peutetre encore dans votre pays, de conduire dans les greniers les petits poulets dans les temps ou les petits papillons commencent a y voler.

pendant qu'on fait cette seconde edition qui aura des augmentations; on imprime un extrait du mesme ouvrage, qui ne contient precisement que ce que la pratique demande qu'on sache ${ }^{92}$.

vous faites trop d'honneur a mon ouvrage sur le insectes en m'en demandant des nouvelles. il y en avoit un volume prest a etre imprimé ${ }^{93}$, lorsque je me suis livré a des distractions qui m'ont mené plus loin que je ne l'avoit prevu, les oiseaux ${ }^{94}$ m'ont seduit et m'ont beaucoup occupé; ils sont une partie de l'histoire naturelle qui a ete trop negligee. des materiaux que j'ai rassembles pour de nouveaux ouvrages, m'ont empeche de faire usage de ceux que j'ai sur les insectes. on ne mesure pas assez ses projets de travaux sur la duree de la vie. pour me faire desirer de revenir bientost aux insectes il me suffist que vous aiez paru vous interesser un peu pour eux.

le respectable Mr. Winslou, que j'aime tendrement, et qui vient diner chez moy une fois la semaine, me parle souvent de vous avec la plus grande admiration, il seroit a souhaiter qu'il eust aussi bien conservé ses oreilles que sa teste. il m'a demande plus d'une fois que lorsque je vous ecrirois je joignisse pour lui les protestations semblables a celles que je vous ferois, de la tres grande estime, du parfait attachement, et du veritable respect avec lequel j'ai l'honneur d'etre

Monsieur

Votre tres humble

et tres obeisant serviteur

de Reaumur

\title{
19.- Réaumur a Morgagni, 2 Settembre 1751
}

a paris ce $2^{\mathrm{e}}$ septembre.

les additions, Monsieur, que j'ai faites a l'art de faire eclorre et elever les oiseaux domestiques, ne sont pas assez considerables pour le rendre digne de paroitre une seconde fois ${ }^{95}$ a vos yeux; et l'extrait de cet ouvrage qui porte le titre de pratique etc. ${ }^{96}$ ne contient qu'une partie de ce qui est dans l'ouvrage. je n'hesite pourtant pas a vous envoyer un exemplaire de l'un et de l'autre; c'est un occasion que je sasis, de vous renouveller les assurances de la tres grande estime, et du parfait et respectueux attachement avec lequel j'ai l'honneur d'etre

Monsieur

\author{
Votre tres humble \\ et tres obeisant serviteur \\ de Reaumur
}

92 Pratique de l'art de faire éclorre et d'élever en toute saison des oiseaux domestiques de toutes especes, Soit par le moyen de la chaleur du fumier, soit par le moyen de celle du feu ordinaire, Paris 1751.

${ }^{93}$ Per gli scritti postumi del Réaumur sugli insetti rimando alla p.12 della cronologia del Torlais citata alla Nota 5.

${ }^{94}$ Sulla raccolta ornitologica del Réaumur è da vedere il capitolo «Le Musée de M. de Réaumur» alle pp.315-345 nell'opera del Torlais citata alla Nota 4.

${ }^{95}$ È la $2^{\text {a }}$ edizione richiamata dalla Nota 89.

${ }_{96}$ È l'opera citata alla Nota 92. 


\section{0.- Morgagni a Réaumur,13 Novembre 1751}

Allo stesso

Oh quanto grazie hò da rendervi per quelle molte che vi siete degnato impartirmi! Primieramente voi aveste la bontà di gradire, di leggere, e di lodare quelle mie Pistole sopra Celso, e Samonico. L'aveste ancora di darmi una gratissima nuova, cioè che della vostra Grand'Opera intorno agl'Insetti avevate già un Volume in pronto per la stampa, e che per ripigliarla, vi compiacevate di far qualche caso anche delle mie premurose istanze, le quali riverentemente vi replico. Ora m'onorate col pregiatissimo Dono sì della nuova edizione dell'Arte di far nascere, ed allevare in qualunque stagione gli uccelli domestici, come della Pratica della medesima, utile altretanto, quanto l'Arte e insieme utile, e dotta, e in oltre accresciuta con l'ultima Memoria, e con le molte, e varie Aggiunte, da me tutte lette con sommo piacere, ed una con tal sentimento d'obbligazione quale è ben dovuto al segnalato Onore che in essa v'è piaciuto di farmi ${ }^{97}$, e che insieme co' vostri libri passerà alla più lontana posterità, di cui sarà il giudizio assai vantaggioso per me, perchè in nissuna cosa saprà ella discostarsi dal vostro. Di questo adunque tanto maggiori grazie vi conserverò sempre nell'animo, quanto meno ve le saprei rendere condegne con le parole.

Quello poi che favoriste di scrivermi per parte dell'ugualmente Cortese, ed Illustre Mr. Winslou accrebbe verso di lui, e di voi le mie obbligazioni. Vi supplico di ringraziarlo molto a mio nome della singolar bontà che hà per me, e d'accertarlo che prego Dio a conservarcelo lungamente a vantaggio della Notomia, della quale non conosco a nostri tempi chi più benemerito sia di lui.

Mi trovo poi anche in necessità di pregarvi a voler fare i miei complimenti al Degnissimo Mr. Morand, e dirgli, che dal ricevere che ora fò per di lui parte il Tomo di cotesta Regia Accademia delle Scienze 1746. ${ }^{98}$ argomento, ch'egli mi avrà già con la stessa bontà mandato anche il Tomo $1745 .{ }^{99} \mathrm{e}$ forse altre cose ancora; le quali con esso Tomo non sò dove, nè presso di chi si possano essere fermate per istrada, giacchè dopo la di lui lettera degli 8. Luglio 1747. sino a questo giorno nissun' altra m'è pervenuta di lui. Arrossisco di recarvi questo incomodo e ve ne domando perdono; ma temendo che le mie lettere a lui dirette si perdano, come le sue; hò creduto necessario di farglielo sapere per vostro mezzo.

E di bel nuovo d'ogni Grazia impartitami ringraziandovi più con l'animo, che con le parole, passo a rassegnarmi con la più grande stima, obbligazione, ed ossequio

Monsieur

Padova 13. Novembre 1751.

\section{1.- Réaumur a Morgagni, 1 Maggio 1752}

a paris ce $1^{\text {er }}$ may 1752

j'ai grand'honte, Monsieur, d'etre encore aujourdui a vous faire mes remerciments de votre tres obligeante letre que je recus vers la fin de l'année derniere. je ne chercherai point a me justifier sur des occupations qui peuvent avoir fait differer ma reponse de quelques

97 Si veda il brano richiamato dalla Nota 91.

98 Che dal frontespizio risulta stampato nel 1751 .

${ }^{99}$ Che dal frontespizio risulta stampato nel 1749. 
semaines; j'aime mieux avouer qu'aiant laisse passer des semaines sans vous ecrire, j'ai insensiblement laissé passer des mois; quand on a commencé a se rendre coupable il est ordinaire de se le rendre de plus en plus. je ne scaurois pourtant assez vous dire combien j'ai été flatté de voir dans la letre qui occasionne les justes reproches que je me fais, que c'est votre cœur qui met le prix a ce que vous recevez de moy; les sentiments ne vous permettent pas de juger mes ouvrages comme vous le feriez s'ils partoient d'une plume qui vous fust indifferente les eloges que vous donnez a de legeres addittions faites a l'art de faire eclorre, et d'elever les oiseaux etc. en sont une preuve incontestable; les miens ne scauroient assurement rien ajouter a une reputation aussi etendue et aussi solidement etablie que la votre mais j'ai ete bien aise de ne pas laisser ignorer au public que je vous devois de la reconnoissance, il m'est glorieux de vous en devoir.

j'aime, Monsieur, Mr. Winslou de tout mon cœur, je n'ai donc eu garde de laisser passer une occasion de lui faire un plaisir tres sensible que je lui ai donné en lui faisant lire l'article de votre letre qui le regarde; il en a ete enchante. mais il pretend que c'est a vous a qui sont justement dus toutes les eloges que vous lui donnez sur les grands services qu'il a rendus a l'Anatomie. il est un de vos plus veritables et plus dignes admirateurs. sa sante est assez bonne, quoiqu'il soit tres delicat. sa teste est toujours la mesme; mais il est d'une surdité qui ne permet pas de lier aucune conversation avec lui; pour peu que j'aie a lui faire entendre des choses un peu longues a dire, je prens le parti de les lui ecrire pour menager ma poitrine. jamais il n'y eust un ame plus pure, plus remplie de candeur, de verité et de modestie que la sienne.

vous auriez du avoir recu l'exemplaire des memoires de l'Academie de l'annee 1745 avant celui de l'annee 1746, c'est par la voye de Mr Morosini, ci devant Ambassadeur de venise a notre cour, que Mr. Morand vous a envoye l'un et l'autre. il lui paroist impossible que le premier se soit egare de maniere a ne se pas retrouver et il ne doute pas qu'il ne vous soit parvenu depuis que vous m'avez ecrit. il a encore a vous faire tenir le volume des mesmes memoires de l'année $1747^{100}$. il m'a charge de vous faire mille et mille compliments des plus sinceres et des plus vifs.

dans notre derniere assemblee publique de l'Academie, je lus un memoire sur une matiere qui ne scauroit vous etre indifferente, c'est un extrait de diverses experiences que j'ai faites sur la maniere dont se fait la digestion dans les oiseaux de differentes classes, qui me paroissent propres a decider les questions qui ont partage les phisiciens sur la maniere dont elle est operee dans toutes les autres especes d'animaux.

ces experiences demontrent que dans touts les oiseaux qui ont un estomach charnu tres compacte, un gesier ${ }^{101}$ que la digestion est l'ouvrage de la trituration; que les dissolvants n'ont aucune part a la division des aliments qui les reduit dans une espece de bouillie.

d'autres experiences demontrent au contraire que dans les oiseaux dont l'estomach est

100 Che dal frontespizio risulta stampato nel 1752.

${ }^{101}$ Sur la digestion des Oiseaux. Premier Mémoire. Expériences sur la manière dont se fait la digestion dans les Oiseaux qui vivent principalement de graines et d'herbes, et dont l'estomac est un gésier, Histoire de l'Académie Royale des Sciences. Année MDCCLII. Avec les Mémoires..., Paris 1756, pp.49-59 della «Histoire» e pp.266-307 dei «Mémoires». 
membraneux ${ }^{102}$ le broyement non contribue en rien a la digestion des aliments; elle y est uniquement faite par un dissolvant. on peut avoir assez de ce dissolvant pour en faire des essais propres a nous faire connoitre la nature, pour le soumetre aux epreuves auxquelles la chimie soumet le liqueurs dont elle veut nous decouvrir les principes. ce sont les oiseaux de proye qui m'ont donne des moyens faciles de multiplier les experiences sur les estomachs membraneux des oiseaux. ceux de proye rejettent ordinairement par le bec au bout de 24 heures les matieres que le leur n'a pu digerer.

mais dans une letre je ne scaurois vous expliquer le detail de mes experiences; le peu que je viens d'en dire sera plus que suffisant pour vous qui scavez voir bien au dela de ce qui est offert a vos yeux.

j'ai l'honneur d'etre avec le plus parfait attachement qu'une grande estime puisse faire naitre et avec respect

Monsieur

Votre tres humble et tres

obeisant serviteur

de Reaumur

\section{Appendice: Le «lunettes en loupe»}

La descrizione delle «lunettes en loupe» richiamata dal Morgagni nella Lettera 5, era stata inclusa dal Réaumur nel Tomo $3^{\circ}$ dei Mémoires, a proposito del pungiglione della mosca galligena dei roseti selvatici, il quale, in posizione di riposo, è arrotolato entro l'addome dell'insetto. Per riuscire ad osservarlo, scrive il Réaumur ${ }^{103}$, bisogna

«tenter une espece de dissection, qui n'est pas aussi difficile qu'on pourroit se l'imaginer. Elle devient même très-simple, si on est muni, comme je le suis, de lunettes aussi fortes que des loupes; alors les yeux ont le secours d'un verre qu'on n'est point obligé de tenir, et les deux mains sont libres ; l'une tient l'insecte pendant que l'autre le disseque. Je profite avec plaisir de cette occasion d'avertir combien des lunettes en loupe peuvent être utiles à ceux qui veulent dissequer des insectes. Mais pour revenir à nos mouches, pendant que j'en tenois une d'une main, l'autre main armée d'une lancette tranchante, ou de ciseaux très-fins, emportoit une très-longue portion des écailles du dos; dès que cette portion étoit emportée, il m'étoit aisé de voir l'aiguillon ${ }^{104}$ qui étoit appliqué auparavant contre la concavité de la partie enlevée.»

Il Réaumur ritorna sulle «lunettes en loupe» (o «lunettes à loupe») nel Tomo $5^{\circ}$ dei Mémoires, a proposito della testa larvale dell'ape ${ }^{105}$ :

"Ces deux crochets ${ }^{106}$ suivent le contour du bout supérieur de la tête; ils se terminent près de la lévre supérieure par une petite pointe écailleuse et jaunâtre. Ils sont si exacte-

${ }^{102}$ Sur la digestion des Oiseaux. Second Mémoire. De la manière dont elle se fait dans l'estomac des Oiseaux de proie. Histoire de l'Académie Royale des Sciences. Année MDCCLII. Avec les Mémoires..., Paris 1756, pp.59-71 della «Histoire» e pp.461-495 dei «Mémoires».

103 Mémoires 3, 487.

104 Pl. 36. fig. 6.

105 Mémoires 5, 579.

106 Pl.36.fig. 9.c,c. 
ment appliqués contre le contour de la tête, qu'il ne seroit pas possible de les y distinguer, si on n'avoit les deux mains libres pendant qu'on les regarde à la loupe; c'est-à-dire, si on n'avoit sur le nez une de ces lunettes à loupe dont nous avons enseigné ailleurs à se servir, qui donnent l'usage d'une main de plus. Swammerdam ${ }^{107}$ à qui ce secours manquoit, avouë naturellement qu'il n'a pû bien voir les parties de la tête de ce ver; et cela, ajoûte-t-il, faute d'une main qui pût les écarter les unes des autres; car l'une de ses mains étoit occupée à tenir le ver, et l'autre à tenir la loupe. Mon nez étant chargé de la loupe, j'ai eu la main nécessaire pour éloigner avec la pointe d'une épingle, un des crochets du contour de la tête contre lequel il étoit appliqué.»

Nel 1765, allorché ricevette la visita di Domenico Cotugno (1736-1822), il Morgagni ricordò il dono delle «lunettes en loupe» e il relativo scambio epistolare col Réaumur, che risalgono, come si è visto, al 1738. Il loro discorso trovasi così fissato nel diario di viaggio del Cotugno ${ }^{108}$ :

«Meckelium ${ }^{109}$ addebat in ea icone ${ }^{110}$ quam de nervis faciei fecit crederemne naturam ubique secutum. Satis artis inquio in minutis illis elucere. Ego quoque sic puto, ait Morgagnus, praecipuos nervos veros esse, reliquos fictos de industria. Vix enim posse videtur ad eam tenuitatem ${ }^{111}$ nervos sequi, et constanter observare.

Incidit hic mentio visionis quam ajebat Morgagnus se specillis raro augere ${ }^{112}$. Qua in re Reaumurium memorabat, qui illa ad insecta pertinentia opera ut edebat mittere ad Morgagnum consuevit : et aliquid quod ad illa attineret quaerebat ex Morgagno. Bis id fecerat Morgagnus. Tertio ut volumen accepit gratias tantum aegit, neque aliud ultra. Instare autem Reaumurius ut aliquid rescriberet. Tum Morgagnus haec. Puto inquit Te Reaumuri illud habere quod ego semper optavi, neque, ut caeteri homines, obtinui. Etenim tres mihi videris habere manus, unam qua insectum tenes, alteram qua secas, tertiam qua microscopium sustines ad videnda tam minuta. Tum Reaumurius respondit, tertiae manus munere non se solum sed et Morgagnum putare eadem ratione fungi. Etenim credebat qua aetate esset jam Morgagnus, uti senes solent, perspicillis uti: quod ut facere aeque posset suo simile ad eum misit perspicillum. Hoc accepisse se Morgagnus fatebatur ita elaboratum ut altero latere acutiorem lentem, minus acutum altero perspicillum haberet; ut quo oculo acutius vellet plus minus videri, obverti lens acutior ei posset.»

107 Jan Swammerdam (1637-80), autore della Historia insectorum generalis (Utrecht 1669) e di Biblia naturae, sive historia insectorum (Leida 1737/8).

108 Dominici Cotunnir, Iter Italicum anni MDCCLXV edito da Luigi Belloni, Memorie dell' Istituto Lombardo - Classe di Lettere 2 d.s.IV (1960) 1-93 (cfr.61-62).

Sui rapporti fra il Cotugno e il Morgagni è da vedere lo scambio epistolare già ricordato nella Nota 10.

109 Johann Friedrich Meckel (1724-74), anatomico a Berlino.

110 Il Morgagni allude alla tavola che il Meckel aveva portato a corredo della «Dissertation anatomique sur les nerfs de la face", Histoire de l'Académie Royale des sciences et belles lettres, Berlino 1751, pp.19-130 dei «Mémoires» della "Classe de philosophie expérimentale».

111 Basti ricordare che i nervi e i loro ramuscoli sono indicati da ben 310 richiami, la cui spiegazione occupa 16 pagine (115-130) a carattere minuto.

112 Sullo stato della vista del Morgagni è da vedere il lavoro qui citato alla Nota 36 . 